\title{
The isotropic XY model on the inhomogeneous periodic chain
}

\author{
J. P. de Lima ${ }^{a, 1}$, T. F. A. Alves ${ }^{b}$, L. L. Gonçalves ${ }^{a, 2, *}$ \\ ${ }^{a}$ Departamento de Física Geral, Instituto de Física, Universidade de São Paulo, \\ C.P.66318, 05315-970, São Paulo, SP, Brazil \\ ${ }^{b}$ Departamento de Física, Universidade Federal do Ceará, Campus do Pici, \\ C.P.6030, 60451-970, Fortaleza, Ceará, Brazil
}

\begin{abstract}
The static and dynamic properties of the isotropic XY-model $(s=1 / 2)$ on the inhomogeneous periodic chain, composed of $N$ segments with $n$ different exchange interactions and magnetic moments, in a transverse field $h$ are obtained exactly at arbitrary temperatures. The properties are determined by introducing the generalized Jordan-Wigner transformation and by reducing the problem to a diagonalization of a finite matrix of $n$-th order. The diagonalization procedure is discussed in detail and the critical behaviour induced by the transverse field, at $T=0$, is presented. The quantum transitions are determined by analyzing the behaviour of the induced magnetization, defined as $(1 / n) \sum_{m=1}^{n} \mu_{m}<S_{j, m}^{z}>$ where $\mu_{m}$ is the magnetic moment at site $m$ within the segment $j$, as a function of the field, and the critical fields determined exactly. The dynamic correlations, $<S_{j, m}^{z}(t) S_{j^{\prime}, m^{\prime}}^{z}(0)>$, and the dynamic susceptibility $\chi_{q}^{z z}(\omega)$ are also obtained at arbitrary temperatures. Explicit results are also presented in the limit $T=0$, where the critical behaviour occurs, for the static susceptibility $\chi_{q}^{z z}(0)$ as a function of the transverse field $h$, and for the frequency dependency of dynamic susceptibility $\chi_{q}^{z z}(\omega)$. Also in this limit, the transverse time-correlation $<S_{j, m}^{x}(t) S_{j^{\prime}, m^{\prime}}^{x}(0)>$, the dynamic and isothermal susceptibilities, $\chi_{q}^{x x}(\omega)$ and $\chi_{T}^{x x}$, are obtained for the transverse field greater or equal than the saturation field.
\end{abstract}

Key words: XY model, quantum transition, inhomogeneous chain PACS: 05.70.Fh, 05.70.Jk, 75.10.Jm, 75.10.Pq

\footnotetext{
* Corresponding author: L. L. Gonçalves. Fax: +55-85-3288-9636

E-mail address:lindberg@fisica.ufc.br

1 On a post-doctoral leave from Departamento de Física, Universidade Federal do Piauí, Campus Ministro Petrônio Portela, 64049-550, Teresina, Piauí, Brazil.

2 On sabbatical leave from: Departamento de Física, Universidade Federal do
} 


\section{Introduction}

Models involving inhomogeneous spin chains have been subject of intensive study in recent years motivated by various reasons. Amongst those, the most remarkable one is the necessity to understand the unusual new properties presented by low dimensional magnetic materials $[1,2,3,4]$ at low temperature, which are described in terms of its many-body behaviour and its quantum transitions [5]. As manifestation of these properties we can mention the appearance of magnetization plateaus as functions of the external magnetic field $[6,7]$, the existence of an energy gap between the ground state and first excited state at zero field [8] and the presence of quantum critical behaviour $[4,9,10]$.

The one-dimensional XY model $(s=1 / 2)$ introduced by Lieb et al. [11] plays an important role in this context since it constitutes one of the few manybody problems which can be exactly solved. The most recent results on the inhomogeneous anisotropic model have been obtained by Derzhko et al.[12] and are restricted to the thermodynamic properties. A good review of the known results is also presented in his work.

For the isotropic model, the thermodynamic properties have also been obtained by Derzhko[13] (see references therein for thermodynamic properties of the alternating chain), and the study of the dynamics and of the quantum critical behaviour has been restricted to the alternating chain $[14,15,16]$, and for the alternating superlattice[17].

In this paper we will also consider the isotropic XY model in a transverse field on the inhomogeneous periodic chain consisting of $N$ unit cells with $n$ sites, which has been studied by Derzkho [13]. Within the cells we can have $n$ different exchange constants as well as magnetic moments, and the model corresponds to an extension of the alternating superlattice. An extensive study of the quantum critical behaviour will be presented and the dynamic properties in the field direction determined for arbitrary temperature. Within a new formalism, we have been able to solve the model exactly, and present new and more general results from those presented in the previous papers $[17,18]$. In particular, we have been able to obtain the dynamic correlation in the xy-plane, at $T=0$, for transverse field greater than the saturation field.

In section 2 we discuss in detail the diagonalization of the model and present the analytical results for the chains composed of cells with two, three and four sites. The excitation spectrum is also obtained, by a different method, in Appendix A, and it is shown under which conditions the gap in the excitation spectrum is suppressed.

Ceará, Campus do Pici, C.P. 6030, 60451-970, Fortaleza, Ceará, Brazil. 


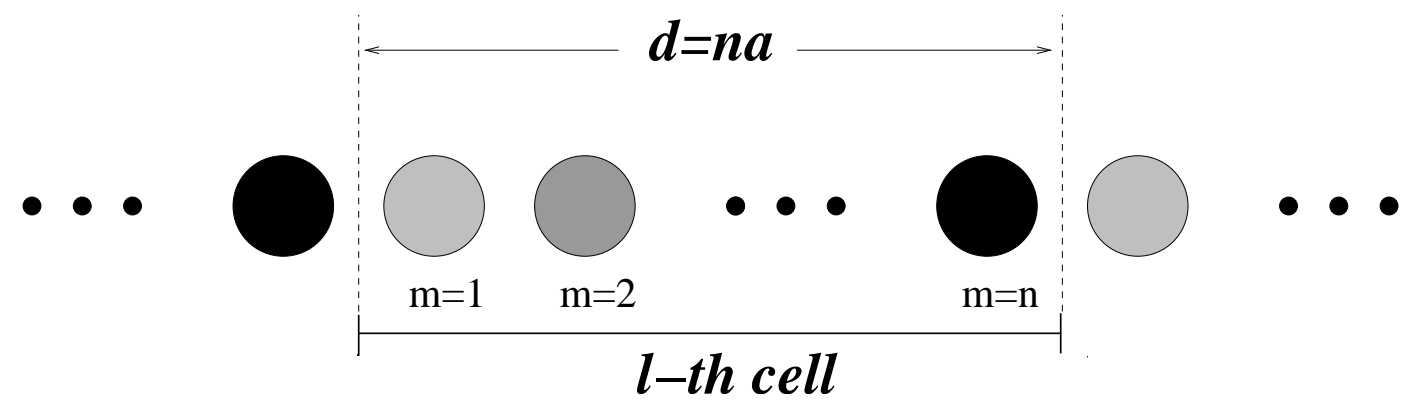

Fig. 1. Unit cell of the periodic chain.

The induced magnetization and the isothermal susceptibility $\chi_{T}^{z z}$ are obtained in section 3 and we also determine the critical fields associated with the quantum second order phase transitions. The static and dynamic correlations in the field direction are obtained in section 4 and, in the section 5, we determine the longitudinal dynamic susceptibility $\chi_{q}^{z z}(\omega)$. The dynamic correlation $\left\langle S_{1, m}^{x}(t) S_{1+l, m^{\prime}}^{x}(0)\right\rangle$ is obtained, at $T=0$ and for external fields greater than the saturation field, in appendix B. Under these conditions, the isothermal and dynamic transverse susceptibility $\chi^{x x}$ are obtained in section 6 , and finally in section 7 we summarize the main results and present the conclusions.

\section{The diagonalization of the Hamiltonian}

We consider the isotropic XY model $(s=1 / 2)$ on the inhomogeneous periodic chain with $N$ cells, $n$ sites per cell, and lattice parameter $a$, in a transverse field, whose unit cell is shown Fig. 1. The Hamiltonian is given by

$$
\begin{aligned}
H= & -\sum_{l=1}^{N}\left\{\sum_{m=1}^{n} \mu_{m} h S_{l, m}^{z}+\sum_{m=1}^{n-1} J_{m}\left[S_{l, m}^{x} S_{l, m+1}^{x}+S_{l, m}^{y} S_{l, m+1}^{y}\right]+\right. \\
& \left.+J_{n} S_{l, n}^{x} S_{l+1,1}^{x}+J_{n} S_{l, n}^{y} S_{l+1,1}^{y}\right\}
\end{aligned}
$$

where the parameters $J_{l, m}$ are the exchange coupling between nearest-neighbour, $\mu_{m}$ the magnetic moments, $h$ the external field and we have assumed periodic boundary conditions. If we introduce the ladder operators

$$
S^{ \pm}=S^{x} \pm i S^{y}
$$

and the generalized Jordan-Wigner transformation[19] 


$$
\begin{aligned}
& S_{l, m}^{+}=\exp \left\{i \pi \sum_{l^{\prime}=1}^{l-1} \sum_{m^{\prime}=1}^{n} c_{l^{\prime}, m^{\prime}}^{\dagger} c_{l^{\prime}, m^{\prime}}+i \pi \sum_{m^{\prime}=1}^{m-1} c_{l, m^{\prime}}^{\dagger} c_{l, m^{\prime}}\right\} c_{l, m}^{\dagger}, \\
& S_{l, m}^{z}=c_{l, m}^{\dagger} c_{l, m}-\frac{1}{2}
\end{aligned}
$$

where $c_{l, m}$ and $c_{l, m}^{\dagger}$ are fermion annihilation and creation operators, we can write the Hamiltonian as[20]

$$
H=H^{+} P^{+}+H^{-} P^{-},
$$

where

$$
\begin{aligned}
H^{ \pm}= & -\sum_{l=1}^{N}\left\{\sum_{m=1}^{n} \mu_{m} h\left(c_{l, m}^{\dagger} c_{l, m}-\frac{1}{2}\right)+\sum_{m=1}^{n-1} \frac{J_{m}}{2}\left(c_{l, m}^{\dagger} c_{l, m+1}+c_{l, m+1}^{\dagger} c_{l, m}\right)\right\}- \\
& -\sum_{l=1}^{N-1} \frac{J_{n}}{2}\left(c_{l, n}^{\dagger} c_{l+1,1}+c_{l+1,1}^{\dagger} c_{l, n}\right) \pm \frac{J_{n}}{2}\left(c_{N, n}^{\dagger} c_{1,1}+c_{1,1}^{\dagger} c_{N, n}\right)
\end{aligned}
$$

and

$$
P^{ \pm}=\frac{I \pm P}{2}
$$

with $P$ given by

$$
P=\exp \left(i \pi \sum_{l=1}^{N} \sum_{m=1}^{n} c_{l, m}^{\dagger} c_{l, m}\right) .
$$

As it is well known $[20,21,22]$, since the operator $P$ commutes with the Hamiltonian, the eigenstates have definite parity, and $P^{-}\left(P^{+}\right)$corresponds to a projector into a state of odd (even) parity.

Introducing periodic and anti-periodic boundary conditions on $c^{\prime} s$ for $H^{-}$and $\mathrm{H}^{+}$respectively, the wave-vectors in the Fourier transform [23],

$$
\begin{aligned}
c_{l, m} & =\frac{1}{\sqrt{N}} \sum_{q} \exp (-i q d l) A_{q, m} \\
A_{q, m} & =\frac{1}{\sqrt{N}} \sum_{l=1}^{N} \exp (i q d l) c_{l, m}
\end{aligned}
$$

are given by $q^{-}=\frac{2 r \pi}{N d}$ for periodic condition and $q^{+}=\frac{\pi(2 r+1)}{N d}$, for anti-periodic condition, with $r=0, \pm 1, \ldots, \pm N / 2$, and $H^{-}$and $H^{+}$can be written in the form

$$
H^{ \pm}=\sum_{q^{ \pm}} H_{q^{ \pm}}
$$

where 


$$
\begin{aligned}
H_{q^{ \pm}}= & -\sum_{m=1}^{n} \mu_{m} h\left(A_{q^{ \pm}, m}^{\dagger} A_{q^{ \pm}, m}-\frac{1}{2}\right)- \\
& -\sum_{m=1}^{n-1} \frac{J_{m}}{2}\left[A_{q^{ \pm}, m}^{\dagger} A_{q^{ \pm}, m+1}+A_{q^{ \pm}, m+1}^{\dagger} A_{q^{ \pm}, m}\right]- \\
& -\frac{J_{n}}{2}\left[A_{q^{ \pm}, n}^{\dagger} A_{q^{ \pm}, 1} \exp \left(-i d q^{ \pm}\right)+A_{q^{ \pm}, 1^{\prime}}^{\dagger} A_{q^{ \pm}, n} \exp \left(i d q^{ \pm}\right)\right] .
\end{aligned}
$$

Although $\mathrm{H}^{-}$and $\mathrm{H}^{+}$do not commute, it can be shown that in the thermodynamic limit all the static properties of the system can be obtained in terms of $\mathrm{H}^{-}$or $\mathrm{H}^{+}$. However, even in this limit, some dynamic properties depend on $H^{-}$and $H^{+}[20,21,22]$. Since

$$
\left[H_{q}, H_{q^{\prime}}\right]=0
$$

where we make the identification $q \equiv q^{ \pm}$, we can diagonalize the Hamiltonian by introducing the canonical transformation

$$
A_{q, m}=\sum_{k=1}^{n} u_{q, k m} \xi_{q, k}, \quad A_{q, m}^{\dagger}=\sum_{k=1}^{n} u_{q, k m}^{*} \xi_{q, k}^{\dagger}
$$

and by imposing the condition

$$
\left[\xi_{q, k}, H_{q}\right]=\varepsilon_{q, k} \xi_{q, k}
$$

which leads, for the coefficients $u_{q, k m}$, to the equation

$$
\mathbf{A}_{q}\left(\begin{array}{c}
u_{q, k 1} \\
u_{q, k 2} \\
\vdots \\
u_{q, k n}
\end{array}\right)=\varepsilon_{q, k}\left(\begin{array}{c}
u_{q, k 1} \\
u_{q, k 2} \\
\vdots \\
u_{q, k n}
\end{array}\right)
$$

where $\mathbf{A}_{q}$ is given by

$$
\mathbf{A}_{q} \equiv-\left(\begin{array}{cccccc}
h_{1} & \frac{J_{1}}{2} & 0 & \cdots & 0 & \frac{J_{n}}{2} \exp (-i q d) \\
\frac{J_{1}}{2} & h_{2} & \frac{J_{2}}{2} & & & 0 \\
0 & \frac{J_{2}}{2} & h_{3} & \frac{J_{3}}{2} & & \vdots \\
\vdots & & \frac{J_{3}}{2} & \ddots & \ddots & 0 \\
0 & & & \ddots & h_{n-1} & \frac{J_{n-1}}{2} \\
\frac{J_{n}}{2} \exp (i q d) & 0 & \cdots & 0 & \frac{J_{n-1}}{2} & h_{n}
\end{array}\right)
$$

and the $u^{\prime} s$ satisfy the orthogonality relations 


$$
\begin{aligned}
& \sum_{m=1}^{n} u_{q, k m} u_{q, k^{\prime} m}^{*}=\delta_{k k^{\prime}}, \\
& \sum_{k=1}^{n} u_{q, k m} u_{q, k m^{\prime}}^{*}=\delta_{m m^{\prime}} .
\end{aligned}
$$

Therefore the Hamiltonian can be written in the diagonal form

$$
H_{q}=\sum_{k} \varepsilon_{q, k}\left(\xi_{q, k}^{\dagger} \xi_{q, k}-\frac{1}{2}\right)
$$

where the spectrum $\varepsilon_{q}$ of $H_{q}$ is determined from the determinantal equation

$$
\operatorname{det}\left(\mathbf{A}_{q}-\varepsilon_{q} \mathbf{I}\right)=0
$$

In passing, we would like to note that for uniform magnetic moments, $\mu_{m} \equiv \mu$, the term $-\sum_{l, m}^{n} \mu h S_{l, m}^{z}$ commutes with the Hamiltonian, and consequently the effect of the field is to shift the spectrum.

By using (16) we can express the operators $\xi^{\prime} s$ in terms of $A^{\prime} s$ which are given by

$$
\xi_{q, k}=\sum_{m=1}^{n} u_{q, k m}^{*} A_{q, m}, \quad \xi_{q, k}^{\dagger}=\sum_{m=1}^{n} u_{q, k m} A_{q, m}^{\dagger},
$$

and from eq.(8) we obtain,

$$
\begin{gathered}
\xi_{q, k}=\frac{1}{\sqrt{N}} \sum_{l=1}^{N} \sum_{m=1}^{n} \exp (i q d l) u_{q, k m}^{*} c_{l, m} \\
\xi_{q, k}^{\dagger}=\frac{1}{\sqrt{N}} \sum_{l=1}^{N} \sum_{m=1}^{n} \exp (-i q d l) u_{q, k m} c_{l, m}^{\dagger}
\end{gathered}
$$

and their inverse, as

$$
\begin{aligned}
c_{l, m} & =\frac{1}{\sqrt{N}} \sum_{q} \sum_{k} \exp (-i q d l) u_{q, k m} \xi_{q, k}, \\
c_{l, m}^{\dagger} & =\frac{1}{\sqrt{N}} \sum_{q} \sum_{k} \exp (i q d l) u_{q, k m}^{*} \xi_{q, k}^{\dagger} .
\end{aligned}
$$

The solution of eqs.(14) and (19) can be obtained analytically for $n \leq 4$, and numerically for $n>4$. In particular, the exact dispersion relations for the cases $n=2,3,4$ are given below.

For $n=2$ is given by

$$
\varepsilon_{q}=-\frac{1}{2}\left(\mu_{1}+\mu_{2}\right) h \pm \frac{1}{2} \sqrt{\left(\mu_{1}-\mu_{2}\right)^{2} h^{2}+J_{1}^{2}+J_{2}^{2}+2 J_{1} J_{2} \cos (2 q)}
$$


and for $n=3$ by [24]

$$
\varepsilon_{q}=-\frac{1}{3}\left(\mu_{1}+\mu_{2}+\mu_{3}\right) h+2 \sqrt{-R} \cos \left(\frac{\theta_{q}+p \pi}{3}\right)
$$

where $p=0,2,4$ and $\theta_{q}=\arccos \left(R_{q} / \sqrt{-R^{3}}\right)$, with $R$ and $R_{q}$ given by

$$
R=\frac{3 a_{2}-a_{1}^{2}}{9}, \quad R_{q}=\frac{9 a_{2} a_{1}-27 a_{q}-2 a_{1}^{3}}{54}
$$

where

$$
\begin{aligned}
& a_{1}=\left(\mu_{1}+\mu_{2}+\mu_{3}\right) h, \\
& a_{2}=\left(\mu_{1} \mu_{2}+\mu_{1} \mu_{3}+\mu_{2} \mu_{3}\right) h^{2}-\frac{1}{4}\left(J_{1}^{2}+J_{2}^{2}+J_{3}^{2}\right), \\
& a_{q}=\mu_{1} \mu_{2} \mu_{3} h^{3}-\frac{1}{4}\left(\mu_{1} J_{2}^{2}+\mu_{2} J_{3}^{2}+\mu_{3} J_{1}^{2}\right) h+\frac{1}{4} J_{1} J_{2} J_{3} \cos (3 q) .
\end{aligned}
$$

For $n=4$, the four branches of the dispersion relation are obtained from the expression [24]

$$
\varepsilon_{q}=-\frac{A}{4}+\frac{\mp \sqrt{A_{1}+2 Y_{q}} \pm \sqrt{-\left(A_{1}+2 Y_{q}\right)-2\left(A_{1} \mp B_{1} / \sqrt{A_{1}+2 Y_{q}}\right)}}{2}
$$

by considering the sign combinations $(+++,---,-+-,+-+)$, and where

$$
\begin{aligned}
& A_{1}=-\frac{3 A^{2}}{8}+B, \quad B_{1}=\frac{A^{3}}{8}-\frac{A B}{2}+C \\
& Y_{q}=-\frac{5}{6} A_{1}-2 \sqrt{\frac{-P_{q}}{3}} \cos \left(\frac{\theta_{q}+2 \pi}{3}\right), \quad \theta_{q}=\arccos \left(\frac{S_{q}}{2} \sqrt{\frac{27}{-P_{q}^{3}}}\right) \\
& P_{q}=-\frac{A_{1}^{2}}{12}-G_{q}, \quad S_{q}=-\frac{A_{1}^{3}}{108}+\frac{A_{1} G_{q}}{3}-\frac{B_{1}^{2}}{8} \\
& G_{q}=-\frac{3 A^{4}}{256}+\frac{B A^{2}}{16}-\frac{A C}{4}+D_{q},
\end{aligned}
$$

and 


$$
\begin{aligned}
A= & \left(\mu_{1}+\mu_{2}+\mu_{3}+\mu_{4}\right) h \\
B= & \left(\mu_{2} \mu_{1}+\mu_{4} \mu_{1}+\mu_{3} \mu_{1}+\mu_{4} \mu_{2}+\mu_{3} \mu_{2}+\mu_{4} \mu_{3}\right) h^{2}- \\
& -\frac{\left(J_{1}^{2}+J_{2}^{2}+J_{3}^{2}+J_{4}^{2}\right)}{4}, \\
C= & \left(\mu_{4} \mu_{3} \mu_{1}+\mu_{3} \mu_{2} \mu_{1}+\mu_{4} \mu_{3} \mu_{2}+\mu_{4} \mu_{2} \mu_{1}\right) h^{3}- \\
& -\frac{\left(J_{4}^{2} \mu_{3}+J_{2}^{2} \mu_{1}+J_{1}^{2} \mu_{3}+J_{4}^{2} \mu_{2}+J_{2}^{2} \mu_{4}+J_{1}^{2} \mu_{4}+J_{3}^{2} \mu_{1}+J_{3}^{2} \mu_{2}\right) h}{4}, \\
D_{q}= & \mu_{4} \mu_{3} \mu_{2} \mu_{1} h^{4}-\frac{\left(J_{4}^{2} \mu_{3} \mu_{2}+J_{3}^{2} \mu_{2} \mu_{1}+J_{2}^{2} \mu_{4} \mu_{1}+J_{1}^{2} \mu_{4} \mu_{3}\right) h^{2}}{4}+ \\
& +\frac{\left(J_{1}^{2} J_{3}^{2}+J_{4}^{2} J_{2}^{2}\right)}{16}-\frac{J_{1} J_{2} J_{3} J_{4}}{8} \cos (4 q) .
\end{aligned}
$$

The excitation spectrum, given by eq.(19), can also be obtained by a transfer matrix technique which leads to a different, but equivalent, expression. This calculation is presented in appendix $\mathrm{A}$, and it is expressed as

$$
\frac{\Im(\omega, h)}{J_{1} J_{2} \ldots J_{n}} \equiv \operatorname{trace}\left[\mathbb{T}_{\text {cell }}(\omega, h)\right]=2 \cos (d q),
$$

where $\mathbb{T}_{\text {cell }}(\omega, h)$ is given by eq.(A.15). Although it is not shown in Appendix A, we have verified that $\Im(\omega, h)$ depends on the square of the exchange constants, $\left\{J_{1}^{2}, J_{2}^{2}, \ldots, J_{n}^{2}\right\}$. This means that the effect of the change of the signs of the $J^{\prime} s$ is to introduce a shift of $\pi / d$ in the spectrum wave-vectors, as can be seen in eq.(30).

As it is also shown in Appendix A, for zero external field, there is no energy gap between the ground state and the first excited state, for $n$ odd and arbitrary $J^{\prime} s$. However, for $n$ even, the gap in the spectrum is only suppressed when the $J^{\prime} s$ satisfy the condition

$$
J_{1} J_{3} \ldots J_{n-1}=J_{2} J_{4} \ldots J_{n}
$$

This latter result can also be obtained from the criticality condition for 1D random Ising model in a transverse field [25] and the equivalence between XY chain and two decoupled transverse field Ising chains[26,27].

The excitation spectrum for a chain with $n=8$, equal and different magnetic moments, is shown in Fig. 2. As can be seen, when the $J^{\prime} s$ satisfy eq.(31) (continuous line) there is no gap at zero field, whereas a gap is present when eq.(31) is not satified (dot-dashed line). Although these results are presented for equal $\mu^{\prime} s$, they are still valid when the $\mu^{\prime} s$ are different.

The dotted line represents the spectrum for nonzero field, different $\mu^{\prime} s$ and the $J^{\prime} s$ also satisfying eq.(31). As can be seen, the spectrum shifts and opens a gap at zero and boundary wave-vectors, which is a consequence of the noncomutativity, in this case, of the field term with the Hamiltonian. 


\section{The induced magnetization and isothermal susceptibility $\chi_{T}^{z z}$}

From eqs.(3) and (22) we can express the local induced magnetization $M_{l, m}^{z}$ as

$$
M_{l, m}^{z} \equiv \mu_{m}\left\langle S_{l, m}^{z}\right\rangle=\frac{\mu_{m}}{N} \sum_{q, k} u_{q, k m}^{*} u_{q, k m} n_{q, k}-\frac{\mu_{m}}{2},
$$

where the occupation number $n_{q, k}$ is given by

$$
n_{q, k}=\frac{1}{1+e^{\beta \varepsilon_{q, k}}},
$$

and the calculation can be done by considering $H=H^{-}[20,21,22]$, since we are interested in the thermodynamic limit.

We can define an average cell magnetization operator in the $z$ direction, $\tau_{l}^{z}$, as

$$
\tau_{l}^{z} \equiv \frac{1}{n} \sum_{m=1}^{n} \mu_{m} S_{l, m}^{z}=\frac{1}{n} \sum_{m=1}^{n} \mu_{m}\left(c_{l, m}^{\dagger} c_{l, m}-\frac{1}{2}\right),
$$

which corresponds to a generalization of the cell spin operator defined in the study of the alternating superlattice[18]. By using eqs. (32) and (34) we can write the induced magnetization per site as

$$
M^{z} \equiv\left\langle\tau_{l}^{z}\right\rangle=\frac{1}{n} \sum_{m=1}^{n} \mu_{m}\left(\frac{1}{N} \sum_{q, k} u_{q, k m}^{*} u_{q, k m} n_{q, k}-\frac{1}{2}\right),
$$

which can be written in the form

$$
M^{z}=-\frac{1}{2 n N} \sum_{q, k, m} \mu_{m} u_{q, k m}^{*} u_{q, k m} \tanh \left(\frac{\beta \varepsilon_{q, k}}{2}\right) .
$$

The isothermal susceptibility can be obtained from the expression

$$
\begin{aligned}
\chi_{T}^{z z} \equiv & \frac{1}{n} \frac{\partial M^{z}}{\partial h}=-\frac{1}{2 n N}\left\{\sum_{q, k, m} \mu_{m} \frac{\partial u_{q, k m}^{*}}{\partial h} u_{q, k m} \tanh \left(\frac{\beta \varepsilon_{q, k}}{2}\right)+\right. \\
& +\sum_{q, k, m} \mu_{m} u_{q, k m}^{*} \frac{\partial u_{q, k m}}{\partial h} \tanh \left(\frac{\beta \varepsilon_{q, k}}{2}\right)+ \\
& \left.+\frac{\beta}{2} \sum_{q, k, m} \mu_{m} u_{q, k m}^{*} u_{q, k m} \operatorname{sech}^{2}\left(\frac{\beta \varepsilon_{q, k}}{2}\right) \frac{\partial \varepsilon_{q, k}}{\partial h}\right\} .
\end{aligned}
$$

At $T=0$, where the system presents quantum transitions, the induced magnetization, obtained from eq. (36) in the limit $T \rightarrow 0$, is given by

$$
M^{z}=-\frac{1}{2 n N} \sum_{q, k, m} \mu_{m} u_{q, k m}^{*} u_{q, k m} \operatorname{sign}\left(\varepsilon_{q, k}\right),
$$


and from eq.(37) we obtain $\chi_{T}^{z z}$, which diverges at the critical fields $h_{c}$. We identify the largest critical field as $h_{s}$, since for $h \geqslant h_{s}$ the induced magnetization is saturated.

For identical magnetic moments, the average cell magnetization operator, $\tau_{l}^{z}$, is proportional to the average cell spin operator, $\frac{1}{n} \sum_{m=1}^{n} S_{l, m}^{z}$, and eqs (36) and (38) can be written, respectively, as

$$
\begin{gathered}
M^{z}=-\frac{1}{2 n N} \sum_{q, k, m} \mu \tanh \left(\frac{\beta \varepsilon_{q, k}}{2}\right), \\
M^{z}=-\frac{1}{2 n N} \sum_{q, k, m} \mu \operatorname{sign}\left(\varepsilon_{q, k}\right),
\end{gathered}
$$

where $\mu_{m}=\mu$ for any $m$.

The results for $M^{z}$ and $\chi_{T}^{z z}$, at $T=0$, are presented in Fig. 3 as functions of the field $h$, for a chain with $n=8$ and identical $\mu^{\prime} s$. The continuous line and the dashed line correspond to the cases where the exchange constants satisfy and do not satisfy the condition shown in eq.(31), respectively. As we have shown for the alternating superlattice [18], the magnetization also presents plateaus which are limited by critical fields $h_{c}$, where the isothermal susceptibility diverges, which correspond to quantum phase transitions induced by the field. The regions of plateaus, which we associate with disordered regions [18], correspond to the gaps in the excitation spectrum, and the critical fields are associated with the zero-energy mode with the wave-vectors $q=0$ and $\pi / d$.

As it can also be seen, when there is no gap in the excitation spectrum at zero field (continuous line), the zero magnetization plateau, which is present when there is a gap (dashed line), is suppressed, and consequently the total number of transitions induced by the field is $n-1$. It should be noted that the local magnetization also presents plateaus and non-analytic behaviour at the critical fields.

For different magnetic moments, the critical fields are obtained as in the previous case, and the results for $M^{z}$ and $\chi_{T}^{z z}$, also at $T=0$, are shown in Fig. 4. The main difference from the previous case is the fact that the magnetization does not present plateaus, and the susceptibility $\chi_{T}^{z z}$ is different from zero at both sides of the transition, although it diverges at one side only. The local magnetization presents similar behaviour as the total magnetization, and there is no suppression of the transition at zero field when there is no gap in the excitation spectrum (continuous line). The regions between two critical fields where the susceptibility $\chi_{T}^{z z}$ is finite correspond to the gaps in the spectrum. As in the case of equal magnetic moments, we associated these regions with disordered regions. 
It should also be noted that for $T>0$, all these transitions are suppressed by the thermal fluctuations.

The exact expressions for the critical fields for $n=2,3$ and 4 , when we have different magnetic moments, can be obtained from the analytic expressions for the spectra given in eqs.(23),(24) and (27), by considering $\varepsilon_{q}=0$ and $q=0$ or $\pi / d$. The explicit results are

$$
\begin{gathered}
h_{c 1}=\frac{\left|J_{1}+J_{2}\right|}{2 \sqrt{\mu_{1} \mu_{2}}}, \quad h_{c 2}=\frac{\left|J_{1}-J_{2}\right|}{2 \sqrt{\mu_{1} \mu_{2}}}, \quad \text { for } n=2[14], \\
h_{c 1}=2 \sqrt{R_{1}^{\prime}} \cos \left(\frac{\theta^{\prime}}{3}\right), \quad h_{c 2}=2 \sqrt{R_{1}^{\prime}}\left|\cos \left(\frac{\theta^{\prime}+2 \pi}{3}\right)\right|, \\
h_{c 3}=2 \sqrt{R_{1}^{\prime}}\left|\cos \left(\frac{\theta^{\prime}+\pi}{3}\right)\right|, \text { for } n=3,
\end{gathered}
$$

where

$$
\begin{array}{r}
\theta^{\prime}=\arccos \left(\frac{R_{2}^{\prime}}{\sqrt{R_{1}^{\prime 3}}}\right) \text { with } 0 \leq \theta^{\prime} / 3 \leq \pi / 2, \\
R_{1}^{\prime}=\frac{J_{1}^{2} \mu_{3}+J_{2}^{2} \mu_{1}+J_{3}^{2} \mu_{2}}{12 \mu_{1} \mu_{2} \mu_{3}}, R_{2}^{\prime}=\frac{J_{1} J_{2} J_{3}}{8 \mu_{1} \mu_{2} \mu_{3}},
\end{array}
$$

and

$$
h_{c 1}=h_{c}^{(+-)}, \quad h_{c 2}=h_{c}^{(++)}, \quad h_{c 3}=h_{c}^{(-+)}, \quad h_{c 4}=h_{c}^{(--)} \text {for } n=4
$$

where

$$
\begin{aligned}
h_{c}^{( \pm \pm)}= & \left\{\frac{1}{8} \frac{\mu_{1} \mu_{2} J_{3}^{2}+\mu_{1} \mu_{4} J_{2}^{2}+\mu_{2} \mu_{3} J_{4}^{2}+\mu_{3} \mu_{4} J_{1}^{2}}{\mu_{1} \mu_{2} \mu_{3} \mu_{4}} \pm\right. \\
& \pm \frac{1}{2 \mu_{1} \mu_{2} \mu_{3} \mu_{4}}\left\{\frac{1}{16}\left(\mu_{1} \mu_{2} J_{3}^{2}+\mu_{1} \mu_{4} J_{2}^{2}+\mu_{2} \mu_{3} J_{4}^{2}+\mu_{3} \mu_{4} J_{1}^{2}\right)^{2}-\right. \\
& \left.\left.-4 \mu_{1} \mu_{2} \mu_{3} \mu_{4}\left[\frac{1}{16}\left(J_{1}^{2} J_{3}^{2}+J_{2}^{2} J_{4}^{2}\right) \pm \frac{1}{8} J_{1} J_{2} J_{3} J_{4}\right]\right\}^{1 / 2}\right\}^{1 / 2} .
\end{aligned}
$$

For arbitrary $n$, the critical fields can also be obtained from the solution of the equation

$$
\frac{\Im(0, h)}{2 J_{1} J_{2} \ldots J_{n}} \pm 2=0 .
$$


For homogeneous magnetic moments we can also obtain from eq. (38), at $T=0$, analytical expressions for the induced magnetization $M^{z}$. For positive values of $J^{\prime} s$, the critical fields satisfy the relation $h_{c i}>h_{c i+1}$ and the induced magnetization, for $n=2$, is given explicitly by

$$
M^{z}= \begin{cases}\frac{\mu}{2} & h \geq h_{c 1} \equiv h_{s} \\ \frac{\mu}{2}-\frac{\mu}{2 \pi} \arccos \Lambda_{2} & h_{c 2} \leq h \leq h_{c 1} \\ 0 & h \leq h_{c 2}\end{cases}
$$

where

$$
\Lambda_{2}=\frac{2 \mu^{2} h^{2}-\frac{1}{2}\left(J_{1}^{2}+J_{2}^{2}\right)}{J_{1} J_{2}},
$$

with $h_{c 1}$ and $h_{c 2}$ given in eq.(41), and for $n=3$ by

$$
M^{z}= \begin{cases}\frac{\mu}{2} & h \geq h_{c 1} \equiv h_{s} \\ \frac{\mu}{2}-\frac{\mu}{3 \pi} \arccos \left(-\Lambda_{3}\right) & h_{c 2} \leq h \leq h_{c 1} \\ \frac{\mu}{6} & h_{c 3} \leq h \leq h_{c 2} \\ \frac{\mu}{6}-\frac{\mu}{3 \pi} \arccos \Lambda_{3} & h \leq h_{c 3}\end{cases}
$$

where

$$
\Lambda_{3}=\frac{-4 \mu^{3} h^{3}+\left(J_{1}^{2}+J_{2}^{2}+J_{3}^{2}\right) \mu h}{J_{1} J_{2} J_{3}}
$$

with $h_{c 1}, h_{c 2}$ and $h_{c 3}$ given in the eq.(42), and finally for $n=4$ by.

$$
M^{z}= \begin{cases}\frac{\mu}{2} & h \geq h_{c 1} \equiv h_{s}, \\ \frac{\mu}{2}-\frac{\mu}{4 \pi} \arccos \Lambda_{4} & h_{c 2} \leq h \leq h_{c 1} \\ \frac{\mu}{4}, & h_{c 3} \leq h \leq h_{c 2} \\ \frac{\mu}{4}-\frac{\mu}{4 \pi} \arccos \left(-\Lambda_{4}\right) & h_{c 4} \leq h \leq h_{c 3} \\ 0 & h \leq h_{c 4},\end{cases}
$$

where $h_{c 1}, h_{c 2}, h_{c 3}$ and $h_{c 4}$ are given in eq.(44), and

$$
\Lambda_{4}=\frac{8 \mu^{4} h^{4}-2\left(J_{3}^{2}+J_{2}^{2}+J_{4}^{2}+J_{1}^{2}\right) \mu^{2} h^{2}}{J_{1} J_{2} J_{3} J_{4}}+\frac{\left(J_{1}^{2} J_{3}^{2}+J_{2}^{2} J_{4}^{2}\right)}{2 J_{1} J_{2} J_{3} J_{4}} .
$$

For arbitrary $n$, between two critical fields, namely, $h_{c j}$ and $h_{c j+1}$, with $h_{c 1} \equiv$ $h_{s}$, a general expression can be obtained for $M_{j}^{z}$ as a function of $h$ and is given by

$$
M_{j}^{z}=M_{p j}^{z}-\frac{\mu}{n \pi} \arccos \left[(-1)^{n+\frac{j+3}{2}} \Lambda_{n}\right],
$$


where $M_{p j}^{z}=\mu[n-2(j-1)] / 2 n$ is the magnetization in the upper plateau and $\Lambda_{n}$ is given by

$$
\Lambda_{n}=\frac{\Im(0, h)}{2 J_{1} J_{2} \ldots J_{n}}
$$

Since the model has azimuthal symmetry, for homogeneous magnetic moments, the values of the induced magnetization per site at the plateaus, $M_{p}$, satisfy the quantization condition [28]

$$
n\left(\mu / 2-M_{p}^{z}\right)=\mu \times \text { integer }
$$

which is verified by the results shown above in eqs.(47-51). For different magnetic moments, although the azimuthal symmetry is preserved, this condition is no more satisfied since the total magnetization operator does not commute with the Hamiltonian. However, it must be noted that eq.(55) is always satisfied provided we replace the induced magnetization per site by the average spin component in the field per site and assume $\mu=1$.

For different $\mu^{\prime} s$, when there are no magnetization plateaus, we can define an order parameter associated with each second order quantum transition given similarly to the case where we have identical $\mu^{\prime} s[17]$ as

$$
\widetilde{M}^{z}=\left|M^{z}-M_{c}^{z}\right|
$$

where $M_{c}^{z}$ is the magnetization at the critical point, and from this we can show that the critical exponent $\beta$, obtained from the scaling relation

$$
\widetilde{M}^{z} \sim\left|h-h_{c}\right|^{\beta}
$$

is equal to $1 / 2$, and the exponent $\gamma$ associated with the isothermal susceptibility, obtained from

$$
\chi_{T}^{z z} \sim\left|h-h_{c}\right|^{-\gamma}
$$

is also equals to $1 / 2$.

For the special case when the $J / s$ satisfy the special condition, eq( 31$)$, the critical exponents $\beta$ and $\gamma$ associated with the transition that occurs at $h=$ 0 are given by 1 and 0 respectively. Therefore, the quantum transitions of the inhomogeneous model, apart from this special point, belong to the same universality class as the ones in the homogeneous model [29]. 


\section{$4 \quad$ Static and dynamic correlations $\left\langle\tau_{l}^{z} \tau_{l+r}^{z}\right\rangle$}

As it has been shown $[20,21,22]$, in the thermodynamic limit, the correlation function $\left\langle S_{l, m}^{z}(t) S_{l+r, m^{\prime}}^{z}(0)\right\rangle$ can be obtained from the expression

$$
\left\langle S_{l, m}^{z}(t) S_{l+r, m^{\prime}}^{z}(0)\right\rangle=\frac{\operatorname{Tr}\left[\exp \left(-\beta H^{-}\right) \exp \left(i H^{-} t\right) S_{l, m}^{z} \exp \left(-i H^{-} t\right) S_{l+r, m^{\prime}}^{z}\right]}{\operatorname{Tr}\left[\exp \left(-\beta H^{-}\right)\right]},
$$

and consequently we can express this correlation in terms of fermion operators. Therefore, the dynamic correlation between the effective spins in the field direction

$$
\left\langle\tau_{l}^{z}(t) \tau_{l+r}^{z}(0)\right\rangle=\frac{1}{n^{2}} \sum_{m, m^{\prime}=1}^{n} \mu_{m} \mu_{m^{\prime}}\left\langle S_{l, m}^{z}(t) S_{l+r, m^{\prime}}^{z}(0)\right\rangle
$$

can be written as

$$
\begin{aligned}
\left\langle\tau_{l}^{z}(t) \tau_{l+r}^{z}(0)\right\rangle= & \frac{1}{n^{2}}\left\{\sum_{m, m^{\prime}=1}^{n} \mu_{m} \mu_{m^{\prime}}\left\langle c_{l, m}^{\dagger}(t) c_{l, m}(t) c_{l+r, m^{\prime}}^{\dagger}(0) c_{l+r, m^{\prime}}(0)\right\rangle-\right. \\
- & \frac{1}{2} \mu_{m} \mu_{m^{\prime}}\left(\left\langle c_{l, m}^{\dagger}(t) c_{l, m}(t)\right\rangle+\left\langle c_{l+r, m^{\prime}}^{\dagger}(0) c_{l+r, m^{\prime}}(0)\right\rangle\right)+ \\
& \left.+\frac{\mu_{m} \mu_{m^{\prime}}}{4}\right\}
\end{aligned}
$$

and by using Wick's theorem [30], we obtain

$$
\left\langle\tau_{l}^{z}(t) \tau_{l+r}^{z}(0)\right\rangle=\frac{1}{n^{2}} \sum_{m, m^{\prime}=1}^{n} \mu_{m} \mu_{m^{\prime}}\left\langle c_{l, m}^{\dagger}(t) c_{l+r, m^{\prime}}(0)\right\rangle\left\langle c_{l, m}(t) c_{l+r, m^{\prime}}^{\dagger}(0)\right\rangle+\left\langle\tau_{l}^{z}\right\rangle^{2}
$$

From eqs.(22), we can write the contractions

$$
\begin{aligned}
& \left\langle c_{l, m}^{\dagger}(t) c_{l^{\prime}, m^{\prime}}(0)\right\rangle=\frac{1}{N} \sum_{q, k} e^{i q d\left(l-l^{\prime}\right)} u_{q, k m}^{*} u_{q, k m^{\prime}} n_{q, k} \exp \left(i \varepsilon_{q, k} t\right) \\
& \left\langle c_{l, m}(t) c_{l^{\prime}, m^{\prime}}^{\dagger}(0)\right\rangle=\frac{1}{N} \sum_{q, k} e^{-i q d\left(l-l^{\prime}\right)} u_{q, k m} u_{q, k m^{\prime}}^{*}\left(1-n_{q, k}\right) \exp \left(-i \varepsilon_{q, k} t\right),
\end{aligned}
$$

and from this equation, we obtain

$$
\begin{aligned}
\left\langle\tau_{l}^{z}(t) \tau_{l+r}^{z}(0)\right\rangle & =\frac{1}{n^{2} N^{2}} \sum_{q, k, m} \sum_{q^{\prime}, k^{\prime}, m^{\prime}} \mu_{m} \mu_{m^{\prime}} u_{q, k m}^{*} u_{q, k m^{\prime}} u_{q^{\prime}, k^{\prime} m} u_{q^{\prime}, k^{\prime} m^{\prime}}^{*} \times \\
& \times n_{q, k}\left(1-n_{q^{\prime}, k^{\prime}}\right) e^{-i d r\left(q-q^{\prime}\right)} e^{i\left(\varepsilon_{q, k}-\varepsilon_{q^{\prime}, k^{\prime}}\right) t}+\left\langle\tau_{l}^{z}\right\rangle^{2},
\end{aligned}
$$


which in the limit $T \rightarrow 0$ can be written as

$$
\begin{aligned}
\left\langle\tau_{l}^{z}(t) \tau_{l+r}^{z}(0)\right\rangle= & \frac{1}{n^{2} N^{2}} \sum_{\substack{q, k \\
\left(\text { for } \varepsilon_{q, k} \leq 0\right)}} \sum_{\substack{q^{\prime}, k^{\prime} \\
\left(\text { for } \varepsilon_{q^{\prime}, k^{\prime}} \geq 0\right)}} \sum_{m, m^{\prime}} \mu_{m} \mu_{m^{\prime}} u_{q, k m}^{*} u_{q, k m^{\prime}} \times \\
& \times u_{q^{\prime}, k^{\prime} m} u_{q^{\prime}, k^{\prime} m^{\prime}}^{*} e^{-i d r\left(q-q^{\prime}\right)} e^{i\left(\varepsilon_{q, k}-\varepsilon_{q^{\prime}, k^{\prime}}\right) t}+\left\langle\tau_{l}^{z}\right\rangle^{2} .
\end{aligned}
$$

The static correlation $\left\langle\tau_{l}^{z} \tau_{l+r}^{z}\right\rangle$ is immediately obtained from the dynamic one by making $t=0$ and we obtain, for finite $T$,

$$
\begin{aligned}
\left\langle\tau_{l}^{z} \tau_{l+r}^{z}\right\rangle & =\frac{1}{n^{2} N^{2}} \sum_{q, m, k} \sum_{q^{\prime}, m^{\prime}, k^{\prime}} \mu_{m} \mu_{m^{\prime}} u_{q, k m}^{*} u_{q, k m^{\prime}} u_{q^{\prime}, k^{\prime} m} u_{q^{\prime}, k^{\prime} m^{\prime}}^{*} \times \\
& \times n_{q, k}\left(1-n_{q^{\prime}, k^{\prime}}\right) e^{-i d r\left(q-q^{\prime}\right)}+\left\langle\tau_{l}^{z}\right\rangle^{2}
\end{aligned}
$$

and

$$
\begin{gathered}
\left\langle\tau_{l}^{z} \tau_{l+r}^{z}\right\rangle=\frac{1}{n^{2} N^{2}} \sum_{\substack{q, k \\
\left(\text { for } \varepsilon_{q, k} \leq 0\right)}} \sum_{\substack{q^{\prime}, k^{\prime} \\
\left(\text { for } \varepsilon_{q^{\prime}, k^{\prime}} \geq 0\right)}} \sum_{m, m^{\prime}} \mu_{m} \mu_{m^{\prime}} u_{q, k m}^{*} u_{q, k m^{\prime}} \times \\
\times u_{q^{\prime}, k^{\prime} m} u_{q^{\prime}, k^{\prime} m^{\prime}}^{*} e^{-i d r\left(q-q^{\prime}\right)}+\left\langle\tau_{l}^{z}\right\rangle^{2}
\end{gathered}
$$

in the limit $T \rightarrow 0$.

For different $\mu^{\prime} s$, we present in Fig. 5, for a chain with $n=8$, at $T=0$, the static correlation as a function of the distance between cells for field values above, below and at a critical point. As expected, for fields below the critical one, which in this case corresponds to the region which contains the divergence of the isothermal susceptibility, the correlation presents an oscillatory behaviour. As in the alternating superlattice [17], the period of the oscillation corresponds to the correlation length and goes to infinity as we approach the critical field, and there is no oscillation in the correlation for fields in the disordered region.

The real and imaginary parts of the dynamic correlation as function of the time, for a chain with the same set of parameters of Fig. 5, are presented in Fig. 6. Differently from the case where we have identical $\mu^{\prime} s$, in the region between two critical fields, where the isothermal susceptibility is finite, the dynamic correlation varies with the field, and for $h \geqslant h_{s}$ is time-independent and equal to $\left\langle\tau_{l}^{z}\right\rangle^{2}$. 


\section{$5 \quad$ Dynamic susceptibility $\chi_{q}^{z z}(\omega)$}

Introducing the time Fourier transform in the dynamic correlation $\left\langle\tau_{l}^{z}(t) \tau_{l+r}^{z}(0)\right\rangle$,

$$
\left\langle\tau_{l}^{z} \tau_{l+r}^{z}\right\rangle_{\omega} \equiv \frac{1}{2 \pi} \int_{-\infty}^{\infty}\left\langle\tau_{l}^{z}(t) \tau_{l+r}^{z}(0)\right\rangle e^{i \omega t} d t
$$

we obtain from eq.(64) the result

$$
\begin{aligned}
\left\langle\tau_{l}^{z} \tau_{l+r}^{z}\right\rangle_{\omega}= & \frac{1}{n^{2} N^{2}} \sum_{q, k, m} \sum_{q^{\prime}, k^{\prime}, m^{\prime}} \mu_{m} \mu_{m^{\prime}} u_{q, k m}^{*} u_{q, k m^{\prime}} u_{q^{\prime}, k^{\prime} m} u_{q^{\prime}, k^{\prime} m^{\prime}}^{*} n_{q, k} \times \\
& \times\left(1-n_{q^{\prime}, k^{\prime}}\right) e^{-i d r\left(q-q^{\prime}\right)} \delta\left(\omega-\varepsilon_{q, k}-\varepsilon_{q^{\prime}, k^{\prime}}\right)+\left\langle\tau_{l}^{z}\right\rangle^{2} \delta(\omega) .
\end{aligned}
$$

By considering the spacial Fourier transform in the field direction,

$$
\left\langle\tau_{q}^{z} \tau_{-q}^{z}\right\rangle_{\omega} \equiv \sum_{r}\left\langle\tau_{l}^{z} \tau_{l+r}^{z}\right\rangle_{\omega} e^{i d r q}
$$

we obtain the correlation as function of $\omega$ and $q$, in the form

$$
\begin{aligned}
\left\langle\tau_{q}^{z} \tau_{-q}^{z}\right\rangle_{\omega} & =\frac{1}{n^{2} N} \sum_{q^{\prime}} \sum_{k, m} \sum_{k^{\prime}, m^{\prime}} \mu_{m} \mu_{m^{\prime}} u_{q^{\prime}, k m}^{*} u_{q^{\prime}, k m^{\prime}} u_{q^{\prime}-q, k^{\prime} m} u_{q^{\prime}-q, k^{\prime} m^{\prime}}^{*} \times \\
& \times n_{q^{\prime}, k}\left(1-n_{q^{\prime}-q, k^{\prime}}\right) \delta\left(\omega+\varepsilon_{q^{\prime}, k}-\varepsilon_{q^{\prime}-q, k^{\prime}}\right)+ \\
& +\left\langle\tau_{l}^{z}\right\rangle^{2} \delta(\omega) \delta_{q, 0} .
\end{aligned}
$$

From this expression we can determine the dynamic susceptibility $\chi_{q}^{z z}(\omega)$, which is given by[31]

$$
\chi_{q}^{z z}(\omega) \equiv-2 \pi\left\langle\left\langle\tau_{q}^{z} ; \tau_{-q}^{z}\right\rangle\right\rangle
$$

where the Green function $\left\langle\left\langle\tau_{q}^{z} ; \tau_{-q}^{z}\right\rangle\right\rangle$ is obtained from

$$
\left\langle\left\langle\tau_{q}^{z} ; \tau_{-q}^{z}\right\rangle\right\rangle=\frac{1}{2 \pi} \int_{-\infty}^{\infty} \frac{\left(1-e^{-\beta \omega}\right)\left\langle\tau_{q}^{z} \tau_{-q}^{z}\right\rangle}{\omega-\omega^{\prime}} d \omega^{\prime}
$$

and we obtain

$$
\begin{aligned}
\chi_{q}^{z z}(\omega) & =-\frac{1}{n^{2} N} \sum_{q^{\prime}} \sum_{k, m} \sum_{k^{\prime}, m^{\prime}} \mu_{m} \mu_{m^{\prime}} u_{q^{\prime}, k m}^{*} u_{q^{\prime}, k m^{\prime}} u_{q^{\prime}-q, k^{\prime} m} u_{q^{\prime}-q, k^{\prime} m^{\prime}}^{*} \times \\
& \times \frac{n_{q^{\prime}, k}-n_{q^{\prime}-q, k^{\prime}}}{\omega+\varepsilon_{q^{\prime}, k}-\varepsilon_{q^{\prime}-q, k^{\prime}}},
\end{aligned}
$$


where we have used the identity

$$
1-n_{q, k}=e^{\beta \varepsilon_{q, k}} n_{q, k}
$$

We can also write $\chi_{q}^{z z}(\omega)$ in the form

$$
\begin{aligned}
\chi_{q}^{z z}(\omega) & =\frac{1}{n^{2} 2 N} \sum_{q^{\prime}} \sum_{k, m} \sum_{k^{\prime}, m^{\prime}} \mu_{m} \mu_{m^{\prime}} u_{q^{\prime}, k m}^{*} u_{q^{\prime}, k m^{\prime}} u_{q^{\prime}-q, k^{\prime} m} u_{q^{\prime}-q, k^{\prime} m^{\prime}}^{*} \times \\
& \times \frac{\tanh \left(\beta \varepsilon_{q^{\prime}, k} / 2\right)-\tanh \left(\beta \varepsilon_{q^{\prime}-q, k^{\prime}} / 2\right)}{\omega+\varepsilon_{q^{\prime}, k}-\varepsilon_{q^{\prime}-q, k^{\prime}}},
\end{aligned}
$$

and from this obtain the static susceptibility $\chi_{0}^{z z}(0)$ which is explicitly given by

$$
\begin{gathered}
\chi_{0}^{z z}(0)=\frac{1}{n^{2} 2 N} \sum_{q^{\prime}} \sum_{\substack{k, k^{\prime} \\
k \neq k^{\prime}}} \sum_{m, m^{\prime}} \mu_{m} \mu_{m^{\prime}} u_{q^{\prime}, k m}^{*} u_{q^{\prime}, k m^{\prime}} u_{q^{\prime}, k^{\prime} m} u_{q^{\prime}, k^{\prime} m^{\prime}}^{*} \times \\
\times \frac{\tanh \left(\beta \varepsilon_{q^{\prime}, k} / 2\right)-\tanh \left(\beta \varepsilon_{q^{\prime}, k^{\prime}} / 2\right)}{\varepsilon_{q^{\prime}, k}-\varepsilon_{q^{\prime}, k^{\prime}}}+ \\
+\frac{\beta}{n^{2} 4 N} \sum_{q^{\prime} k} \sum_{m, m^{\prime}} \mu_{m} \mu_{m^{\prime}} u_{q^{\prime}, k m}^{*} u_{q^{\prime}, k m^{\prime}} u_{q^{\prime}, k m} u_{q^{\prime}, k m^{\prime}}^{*} \operatorname{sech}^{2}\left(\beta \varepsilon_{q^{\prime}, k} / 2\right) .
\end{gathered}
$$

As in the uniform model [32], it can be shown from the previous expression that $\chi_{0}^{z z}(0)$ is equal to the isothermal susceptibility $\chi_{T}^{z z}$ given in eq.(37).

The real and imaginary parts of $\chi_{q}^{z z}(\omega)$, as usual, are obtained by considering $\chi_{q}^{z z}(\omega-i \epsilon)$ in the limit $\epsilon \rightarrow 0$ in eq.(76) and are given by

$$
\begin{aligned}
R e \chi_{q}^{z z}(\omega)= & \frac{1}{n^{2} 2 N} P \sum_{q^{\prime}} \sum_{k, m} \sum_{k^{\prime}, m^{\prime}} \mu_{m} \mu_{m^{\prime}} u_{q^{\prime}, k m}^{*} u_{q^{\prime}, k m^{\prime}} u_{q^{\prime}-q, k^{\prime} m} u_{q^{\prime}-q, k^{\prime} m^{\prime}}^{*} \times \\
& \times \frac{\tanh \left(\beta \varepsilon_{q^{\prime}, k} / 2\right)-\tanh \left(\beta \varepsilon_{q^{\prime}-q, k^{\prime}} / 2\right)}{\omega+\varepsilon_{q^{\prime}, k}-\varepsilon_{q^{\prime}-q, k^{\prime}}}
\end{aligned}
$$

where $P$ denotes Cauchy principal value, and

$$
\begin{aligned}
\operatorname{Im}_{\chi_{q}}^{z z}(\omega)= & \frac{\pi}{n^{2} 2 N} \sum_{q^{\prime}} \sum_{k, m} \sum_{k^{\prime}, m^{\prime}} \mu_{m} \mu_{m^{\prime}} u_{q^{\prime}, k m}^{*} u_{q^{\prime}, k m^{\prime}} u_{q^{\prime}-q, k^{\prime} m} u_{q^{\prime}-q, k^{\prime} m^{\prime}}^{*} \times \\
& \times\left(\tanh \left(\beta \varepsilon_{q^{\prime}, k} / 2\right)-\tanh \left(\beta \varepsilon_{q^{\prime}-q, k^{\prime}} / 2\right)\right) \times \\
& \times \delta\left(\omega+\varepsilon_{q^{\prime}, k}-\varepsilon_{q^{\prime}-q, k^{\prime}}\right) .
\end{aligned}
$$

At $T=0$, these results, as function of $\omega$, are shown in Fig. 7, for $n=4$ and different wave-vectors for a field in the disordered region. For any wave-vector 
there are the same number of bands in the imaginary part of the susceptibility, and this number depends on the size of the unit cell and on the values of the parameters.

In Fig. 8 it is shown the static susceptibility, $\chi_{q}^{z z}(0)$, at $T=0$, for the same lattice parameters of Fig. 7, as a function of the field. For $q=0$, since it is identical to the isothermal susceptibility, it diverges at the critical fields. The singularities at non-zero and at the Brillouin zone boundary wave-vectors can be associated with critical points, whereas the ones for different wave-vectors are related to oscillations of the spin correlations and can also be associated with the unstable critical points present in the study of the system within the real space renormalization group [33].

\section{$6 \quad \mathbf{T}=0$ isothermal and dynamic susceptibilities $\chi_{T}^{x x}$ and $\chi_{q}^{x x}(\omega)$}

At $T=0$, and for $\mathrm{h} \geq h_{s}$, we can obtain from the eq.(B.8) the time Fourier transform of dynamic correlation $\left\langle S_{1, m}^{x}(t) S_{1+r, m^{\prime}}^{x}(0)\right\rangle$, which is given by

$$
\left\langle S_{1, m}^{x} S_{1+r, m^{\prime}}^{x}\right\rangle_{\omega}=\frac{1}{2 \pi} \int_{-\infty}^{\infty} \exp (i \omega t)\left\langle S_{1, m}^{x}(t) S_{1+r, m^{\prime}}^{x}(0)\right\rangle d t
$$

and can be written as

$$
\left\langle S_{1, m}^{x} S_{1+r, m^{\prime}}^{x}\right\rangle_{\omega}=\frac{(-1)^{n r+m^{\prime}-m}}{4 N} \sum_{q, k} \exp (-i q d r) u_{q, k m}^{*} u_{q k m^{\prime}} \delta\left(\omega+\varepsilon_{q, k}\right) .
$$

Following Gonçalves [34], we can write the local dynamic susceptibility $\chi^{x x}\left(\omega, r, m, m^{\prime}\right)$ as

$$
\chi^{x x}\left(\omega, r, m, m^{\prime}\right)=-\lim _{\beta \rightarrow \infty} \int_{-\infty}^{\infty} \frac{[1-\exp (-\beta \omega)]\left\langle S_{1, m}^{x} S_{1+r, m^{\prime}}^{x}\right\rangle_{\omega}}{\omega-\omega^{\prime}} d \omega^{\prime},
$$

since $\varepsilon_{q, k} \leq 0$, and by using eq.(81) we obtain

$$
\chi^{x x}\left(\omega, r, m, m^{\prime}\right)=-\frac{(-1)^{n r+m^{\prime}-m}}{4 N} \sum_{q, k} \frac{\exp (-i q d r) u_{q, k m}^{*} u_{q k m^{\prime}}}{\omega+\varepsilon_{q, k}} .
$$

The susceptibility associated with the cell effective spin in the $x$ direction is given by

$$
\chi^{x x}(\omega, r)=\sum_{m, m^{\prime}} \chi^{x x}\left(\omega, r, m, m^{\prime}\right)
$$

and by using eq.(83) we can write

$$
\chi^{x x}(\omega, r)=-\frac{(-1)^{n r}}{4 N} \sum_{q, k, m, m^{\prime}}(-1)^{m^{\prime}-m} \frac{\exp (-i q d r) u_{q, k m}^{*} u_{q, k m^{\prime}}}{\omega+\varepsilon_{q, k}}
$$


and from this the final result

$$
\chi_{q}^{x x}(\omega)=-\sum_{k, m, m^{\prime}} \frac{(-1)^{m-m^{\prime}} u_{q^{\prime}, k m}^{*} u_{q^{\prime}, k m^{\prime}}}{4\left(\omega+\varepsilon_{q^{\prime}, k}\right)},
$$

where

$$
q^{\prime}= \begin{cases}q & \text { for } n \text { even } \\ q-\frac{\pi}{d} & \text { for } n \text { odd }\end{cases}
$$

From eq.(86) by considering the $\chi_{q}^{x x}(\omega-i \epsilon)$ in the limit $\epsilon \rightarrow 0$ we can obtain immediately the real and imaginary parts which are given by

$$
\operatorname{Re} \chi_{q}^{x x}(\omega)=-P \sum_{k, m, m^{\prime}} \frac{(-1)^{m-m^{\prime}} u_{q^{\prime}, k m}^{*} u_{q^{\prime}, k m^{\prime}}}{4\left(\omega+\varepsilon_{q^{\prime}, k}\right)}
$$

where $P$ denotes Cauchy principal value, and

$$
\operatorname{Im} \chi_{q}^{x x}(\omega)=-\frac{\pi}{4} \sum_{k, m, m^{\prime}}(-1)^{m-m^{\prime}} u_{q^{\prime}, k m}^{*} u_{q^{\prime}, k m^{\prime}} \delta\left(\omega+\varepsilon_{q^{\prime}, k}\right) .
$$

We can also obtain, in the limit considered, the local isothermal susceptibility from the expression [35]

$$
\chi_{T}^{x x}\left(r, m, m^{\prime}\right)=\lim _{\beta \rightarrow \infty} \int_{0}^{\beta}\left\langle S_{1, m}^{x}(-i \lambda) S_{1+r, m^{\prime}}^{x}(0)\right\rangle d \lambda,
$$

which, from eq.(B.8), can be written as

$$
\chi_{T}^{x x}\left(r, m, m^{\prime}\right)=-\frac{(-1)^{n r+m^{\prime}-m}}{4 N} \sum_{q, k} \frac{\exp (-i q d r) u_{q, k m}^{*} u_{q, k m^{\prime}}}{\varepsilon_{q, k}} .
$$

From this result we can immediately conclude that the isothermal susceptibility, $\chi_{T}^{x x}=\sum_{r, m, m^{\prime}} \chi_{T}^{x x}\left(r, m, m^{\prime}\right)$, is equal to the static one, $\chi_{q}^{x x}(\omega)$, in the limit $q \rightarrow 0$.

For equal magnetic moments, $n=8$ and the exchange parameters of Fig. 3(a), the real and imaginary parts of the dynamic correlations $\left\langle S_{1, m}^{x}(t) S_{1+r, m^{\prime}}^{x}(0)\right\rangle$ and $\left\langle S_{1, m}^{x}(t) S_{1+r, m^{\prime}}^{x}(0)\right\rangle$ are shown in Figs. 9 and 10 respectively, for various magnetic fields. For large $t$, these correlations factorize and tend to zero, since the average $\left\langle S_{l, m}^{x}\right\rangle \rightarrow 0$.

Due to the simple form of the dynamic susceptibility, eq.(86 ), it presents poles of order one at the excitation energies. This means that the imaginary part corresponds to a set of delta functions for a given wave-vector.

In Fig. 11 we present the static susceptibility $\chi_{q}^{x x}(\omega)$, at $T=0$, as a function of the field for a chain with $n=8$. For the ferromagnetic case shown in Fig. 
11(a) it diverges at the critical field for $q=0$ only. On the other hand, if we change the sign of a single exchange interaction, it diverges for $q$ at the zone boundary, namely, $q=\pi / 8$. This is shown in Fig. 11(b), and means that the system in terms of cells has an antiferromagnetic behaviour. Although it is not presented, it can be shown that the order ferro (antiferro) is associated to the sign $+(-)$ of the product of the exchange interactions within the cells.

\section{Conclusions}

We have considered in this work the isotropic XY model on the inhomogeneous periodic chain with $N$ cells, $n$ sites per cell. The exact solution has been obtained in the general case, where we have $n$ different exchange constants and magnetic moments, and for arbitrary temperatures.

At $T=0$, for equal magnetic moments, we have shown that the induced magnetization, as a function of the field, presents plateaus which satisfy the quantization condition shown in eq. (55), and that the isothermal susceptibility $\chi_{T}^{z z}$ is zero within the plateaus and diverges at the limiting fields which characterize the multiple second order quantum transitions. On the other hand, $f$ or different magnetic moments, although the system presents multiple quantum transitions, the induced magnetization plateaus are suppressed and the isothermal susceptibility, which also diverges at the critical points, is always different from zero. In both cases, the disordered phases correspond to the gaps in the excitation spectrum and to the regions where $\chi_{T}^{z z}$ is finite, which are identical to the plateaus for the case of identical magnetic moments. For $n=1,2,3$, explicit analytical expressions are presented for the critical fields, in the general case, and for the induced magnetization when we have identical magnetic moments.

The number of transitions, for different $\mu^{\prime} s$, is always equal to $n$, even for the special case where the exchange constants, for $n$ even, satisfy the condition presented in eq.(31), where at zero field there is no gap between the ground state and the first excited state. It should be noted that in this case, $\chi_{T}^{z z}$ diverges at both sides of the zero field transition and that, for identical $\mu^{\prime} s$, this transition is suppressed.

The critical exponents are identical to those of the uniform model, $\alpha=1 / 2$, $\beta=1 / 2, \gamma=1 / 2$, and for the special point at zero field $\alpha=0, \beta=1, \gamma=0$, and naturally they satisfy the Rushbrook relation $\alpha+2 \beta+\gamma=2$ (see e.g. ref.[36]). It should be noted that in these transitions $\alpha=\gamma$ [37].

The correlation length, which corresponds to the period of the oscillation of the static correlation, $\left\langle\tau_{l}^{z} \tau_{l+r}^{z}\right\rangle$, as in the homogeneous chain and alternat- 
ing superlattice [17], diverges at the critical points irrespective of the values of the magnetic moments. On the other hand, the dynamic correlation $\left\langle\tau_{l}^{z}(t) \tau_{l+r}^{z}(0)\right\rangle$, which is independent of the field in the plateaus regions for equal magnetic moments, is field dependent when we have different magnetic moments.

The isothermal susceptibility $\chi_{T}^{z z}$ is equal to the the static one, $\chi_{0}^{z z}(0)$, at any temperature. Independently of the value of the field and of the magnetic moments, the imaginary part of the dynamic susceptibility $\chi_{q}^{z z}(\omega)$ presents several bands whose number does not depend on $q$. As in the alternating superlattice, the discontinuities of the imaginary part at the band edges correspond to singularities in the real part. Associated with the critical behaviour are the descontinuities which occur at zero or at the Brillouin zone boundary wave-vectors, whereas those that occur for other values of the wave-vectors are related to unstable critical points [33].

At $T=0$ and for fields greater than the saturation field, we obtained the dynamic correlation $\left\langle S_{1, m}^{x}(t) S_{1+r, m^{\prime}}^{x}(0)\right\rangle$ and the isothermal and wave-vector dynamic susceptibilities in the $x$ direction. We have shown that the isother-

mal susceptibility $\chi_{T}^{x x}$ is identical to the static one $\chi_{0}^{z z}(0)$, and that in terms of the magnetization of the cells the system presents a ferromagnetic or antiferromagnetic order depending on the sign of the product of the exchange interactions within the cells.

\section{Acknowledgements}

The authors would like to thank the Brazilian agencies CNPq, Capes and Finep for partial financial support. They would also like to thank Prof. S. R. A. Salinas for his hospitality during their stay at the Departamento de Física Geral, Instituto de Física, Universidade de São Paulo, and Dr. A. P. Vieira for valuable discussions. 


\section{A Transfer matrix technique: excitation spectrum}

The excitation spectrum of $H_{q}$,

$$
\begin{aligned}
H_{q}= & -\sum_{m=1}^{n} \mu_{m} h\left(A_{q, m}^{\dagger} A_{q, m}-\frac{1}{2}\right)- \\
& -\sum_{m=1}^{n-1} \frac{J_{m}}{2}\left[A_{q, m}^{\dagger} A_{q, m+1}+A_{q, m+1}^{\dagger} A_{q, m}\right]- \\
& -\frac{J_{n}}{2}\left[A_{q, n}^{\dagger} A_{q, 1} \exp (-i q d)+A_{q, 1}^{\dagger} A_{q, n} \exp (i q d)\right],
\end{aligned}
$$

given in eq.(19), can also be obtained by using a transfer matrix method. By introducing the equation of motion for the operators $A_{q, m}$

$$
\dot{A}_{q, m}=\frac{1}{i}\left[A_{q, m}, \sum_{q^{\prime}} H_{q^{\prime}}\right]
$$

and assuming the time-evolution given by

$$
A_{q, m}(t)=A_{q, m} \exp (-i \omega t),
$$

we obtain the system of equations

$$
\begin{aligned}
\left(\omega+\mu_{1} h\right) A_{q, 1} & =-\frac{J_{1}}{2} A_{q, 2}-\frac{J_{n} \exp (i q d)}{2} A_{q, n}, \\
\left(\omega+\mu_{2} h\right) A_{q, 2} & =-\frac{J_{2}}{2} A_{q, 3}-\frac{J_{1}}{2} A_{q, 1}, \\
\vdots & \\
\left(\omega+\mu_{m-1} h\right) A_{q, m-1} & =-\frac{J_{m-1}}{2} A_{q, m}-\frac{J_{m-2}}{2} A_{q, m-2}, \\
\left(\omega+\mu_{m} h\right) A_{q, m} & =-\frac{J_{m}}{2} A_{q, m+1}-\frac{J_{m-1}}{2} A_{q, m-1}, \\
\left(\omega+\mu_{m+1} h\right) A_{q, m+1} & =-\frac{J_{m+1}}{2} A_{q, m+2}-\frac{J_{m}}{2} A_{q, m}, \\
\vdots & \\
\left(\omega+\mu_{n-1} h\right) A_{q, n-1} & =-\frac{J_{n-1}}{2} A_{q, n}-\frac{J_{n-2}}{2} A_{q, n-2}, \\
\left(\omega+\mu_{n} h\right) A_{q, n} & =-\frac{J_{n} \exp (-i q d)}{2} A_{q, 1}-\frac{J_{n-1}}{2} A_{q, n-1},
\end{aligned}
$$




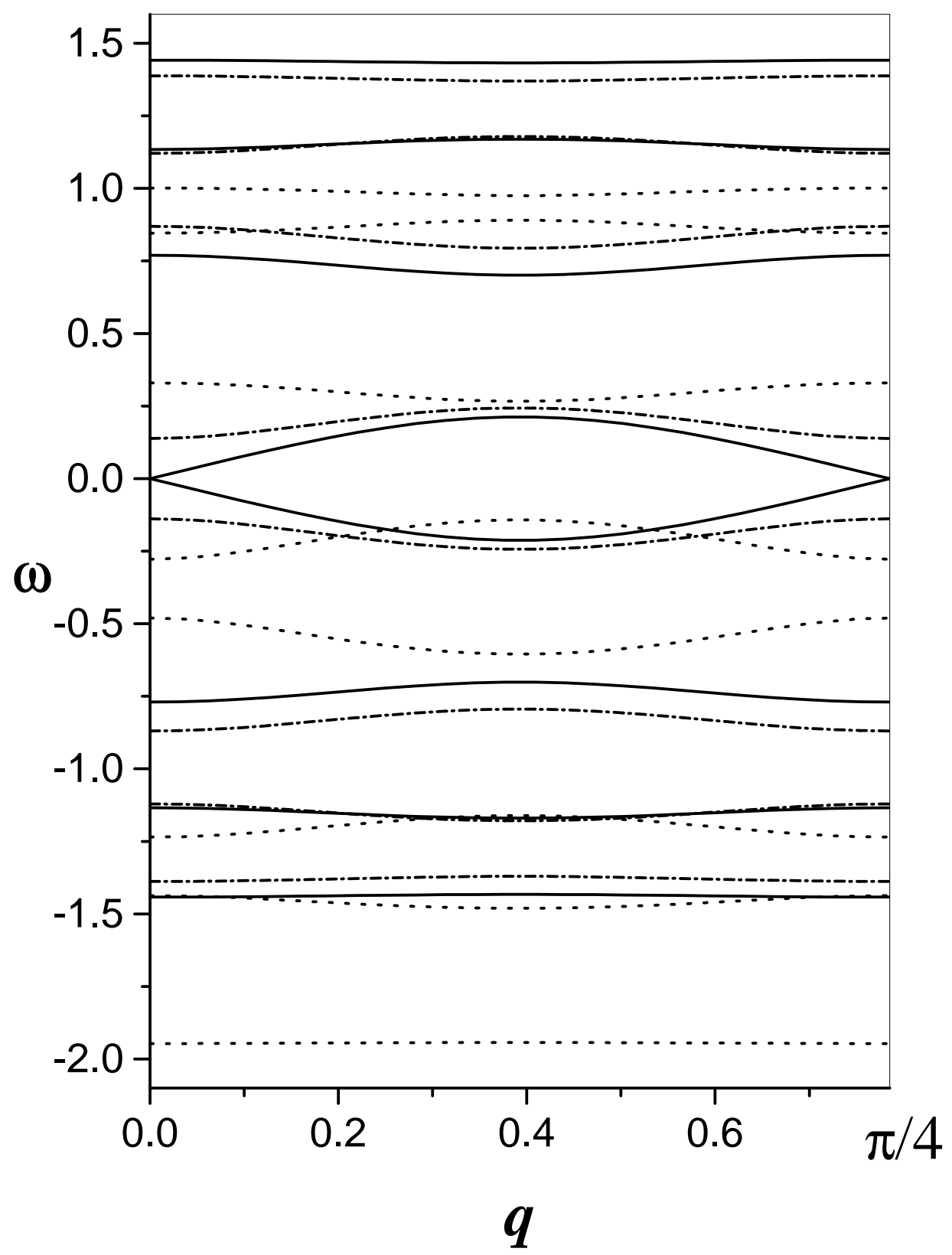

Fig. 2. Excitation spectrum for $n=8, J_{1}=1, J_{2}=2, J_{3}=1.5, J_{4}=5 / 3, J_{5}=2 / 3$, $J_{6}=3 / 5, J_{7}=2, J_{8}=1$ for $\mu_{1}=\ldots .=\mu_{8}=1$ and $h=0$ (continuous line) and for $\mu_{1}=3, \mu_{2}=4, \mu_{3}=\mu_{5}=\mu_{7}=1.5, \mu_{4}=2.5, \mu_{6}=\mu_{8}=1$ and $h=0.2$ (dotted line), and for $J_{1}=2, J_{2}=1, J_{3}=1.5, J_{4}=5 / 3, J_{5}=2 / 3, J_{6}=3 / 5, J_{7}=2$, $J_{8}=1, \mu_{1}=\ldots .=\mu_{8}=1$ and $h=0$ (dot-dashed line). 
(a)

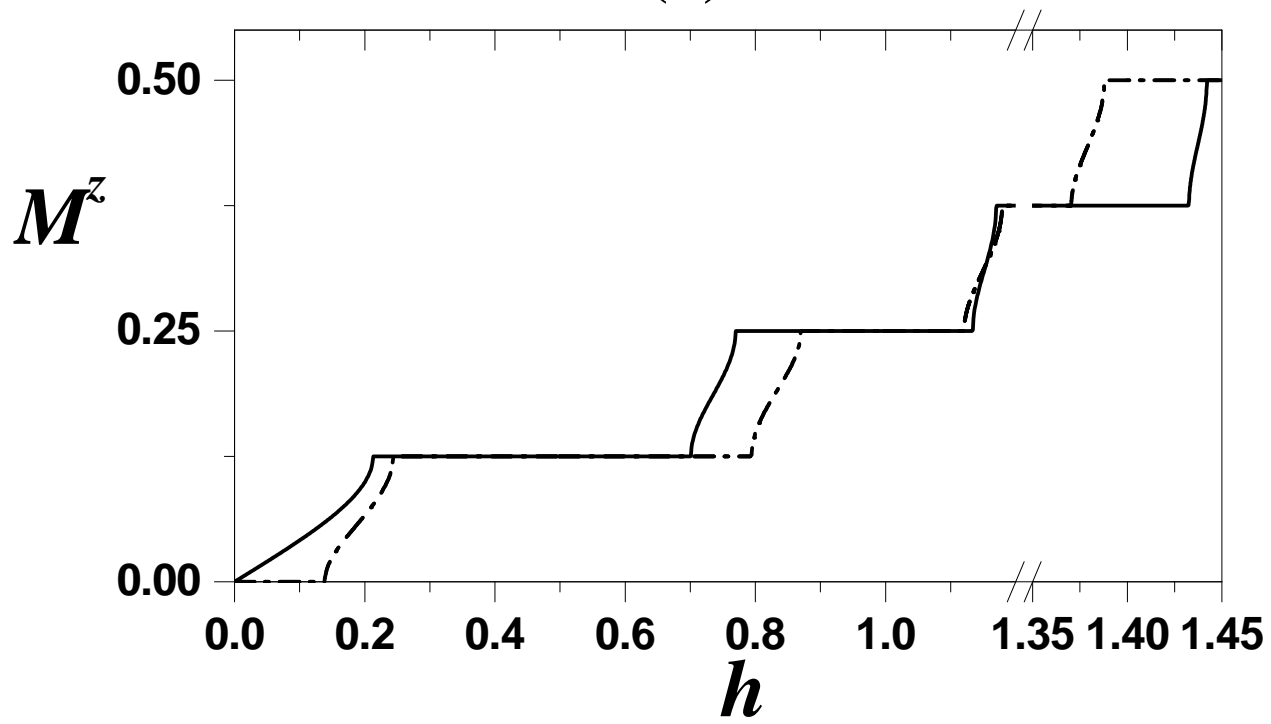

(b)

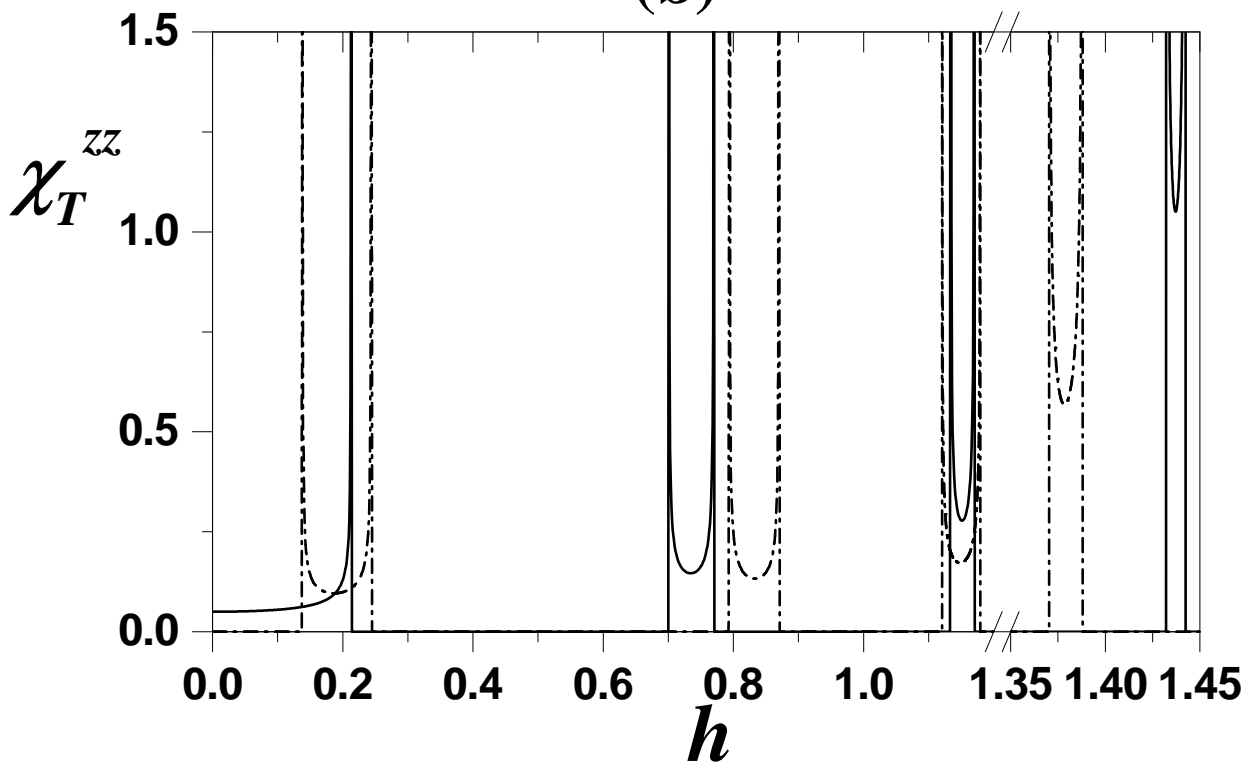

Fig. 3. (a) Magnetization $M^{z}$ and (b) isothermal susceptibility $\chi_{T}^{z z}$,at $T=0$ and for $n=8, \mu_{1}=\ldots=\mu_{8}=1$, as functions of the uniform field for $J_{1}=1, J_{2}=2$, $J_{3}=1.5, J_{4}=5 / 3, J_{5}=2 / 3, J_{6}=3 / 5, J_{7}=2, J_{8}=1$ (continuous line) and $J_{1}=2, J_{2}=1, J_{3}=1.5, J_{4}=5 / 3, J_{5}=2 / 3, J_{6}=3 / 5, J_{7}=2, J_{8}=1$ (dashed line). 
(a)

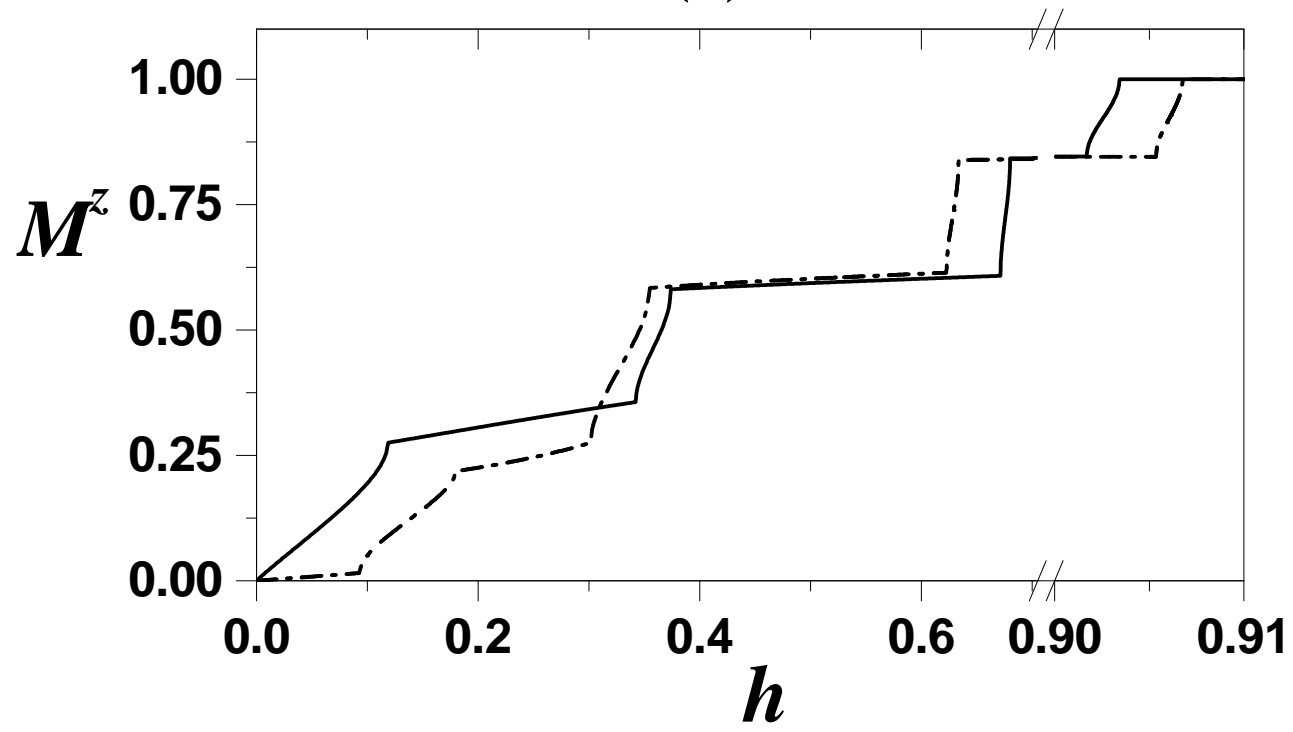

(b)

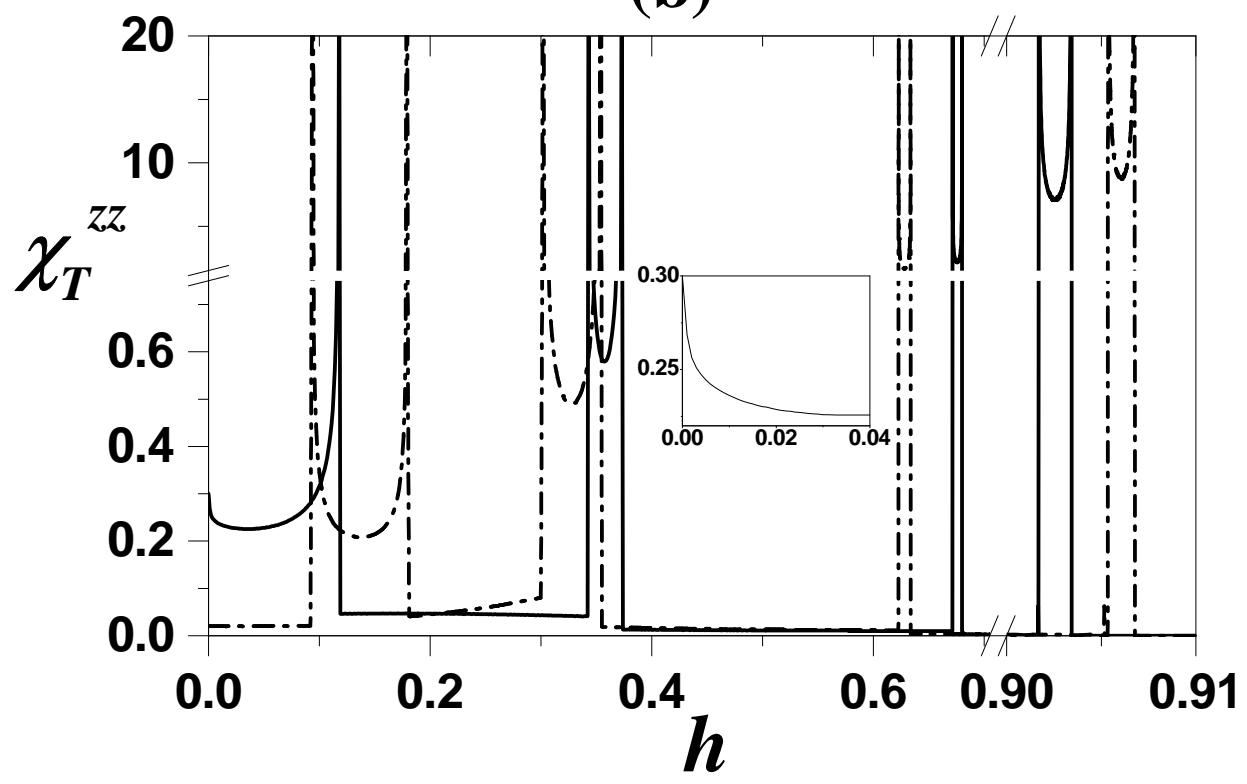

Fig. 4. (a) Magnetization $M^{z}$ and (b) isothermal susceptibility $\chi_{T}^{z z}$, at $T=0$ and $n=8, \mu_{1}=3, \mu_{2}=4, \mu_{3}=\mu_{5}=\mu_{7}=1.5, \mu_{4}=2.5, \mu_{6}=\mu_{8}=1$, as functions of the uniform field for $J_{1}=1, J_{2}=2, J_{3}=1.5, J_{4}=5 / 3, J_{5}=2 / 3, J_{6}=3 / 5$, $J_{7}=2, J_{8}=1$ (continuous line) and $J_{1}=2, J_{2}=1, J_{3}=1.5, J_{4}=5 / 3, J_{5}=2 / 3$, $J_{6}=3 / 5, J_{7}=2, J_{8}=1$ (dashed line). 


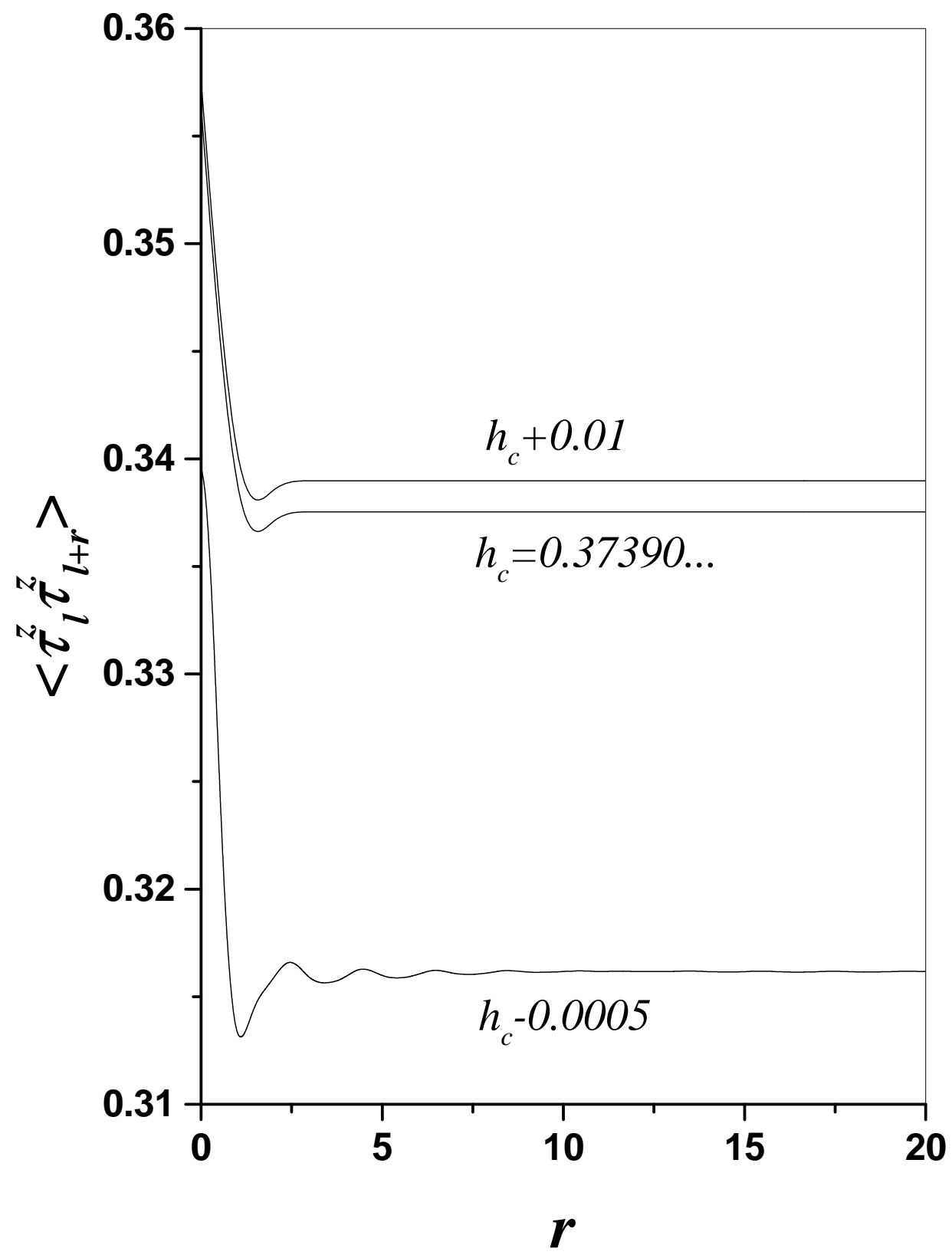

Fig. 5. Static correlation function $\left\langle\tau_{l}^{z} \tau_{l+r}^{z}>\right.$ as a function of $r$ (distance between cells), at $T=0$, for $n=8, J_{1}=1, J_{2}=2, J_{3}=1.5, J_{4}=5 / 3, J_{5}=2 / 3, J_{6}=3 / 5$, $J_{7}=2, J_{8}=1, \mu_{1}=3, \mu_{2}=4, \mu_{3}=\mu_{5}=\mu_{7}=1.5, \mu_{4}=2.5, \mu_{6}=\mu_{8}=1$ for values of the field near and at the critical field. 



Fig. 6. The real and imaginary parts of the correlation function $\left\langle\tau_{l}^{z}(t) \tau_{l+1}^{z}(0)\right\rangle$, as functions of time, at $T=0$, for $n=8, J_{1}=1, J_{2}=2, J_{3}=1.5, J_{4}=5 / 3$, $J_{5}=2 / 3, J_{6}=3 / 5, J_{7}=2, J_{8}=1, \mu_{1}=3, \mu_{2}=4, \mu_{3}=\mu_{5}=\mu_{7}=1.5, \mu_{4}=2.5$, $\mu_{6}=\mu_{8}=1$, for values of the field near and at the critical field. 

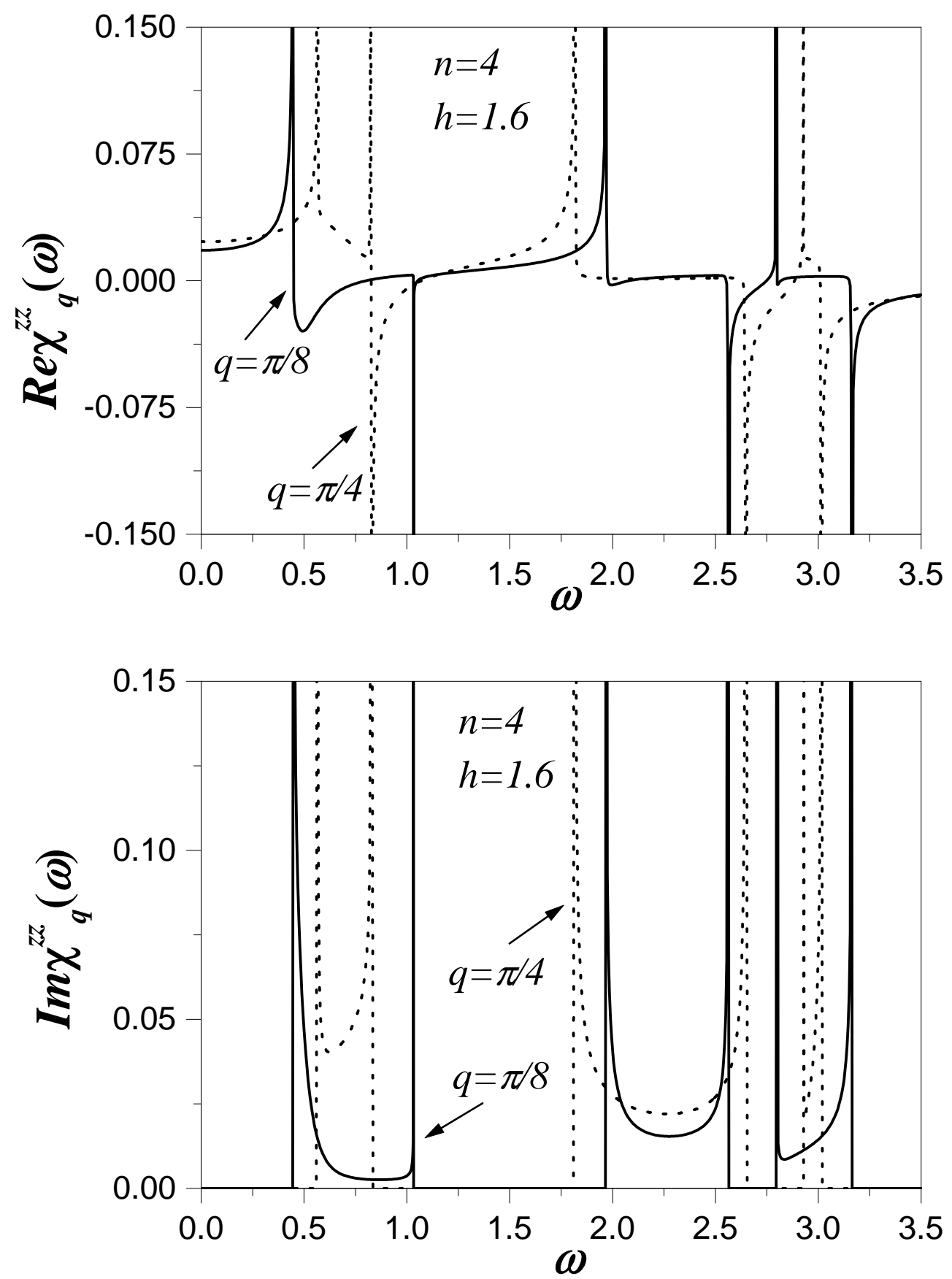

Fig. 7. The real and imaginary parts of the dynamic susceptibility in the field direction, $\chi_{q}^{z z}(\omega)$, at $T=0$ as a function of frequency for $n=4, J_{1}=1, J_{2}=2$, $J_{3}=1.5, J_{4}=5 / 3, \mu_{1}=\mu_{3}=1, \mu_{2}=\mu_{4}=0.5$ and $h=1$,for different values of $q$. 


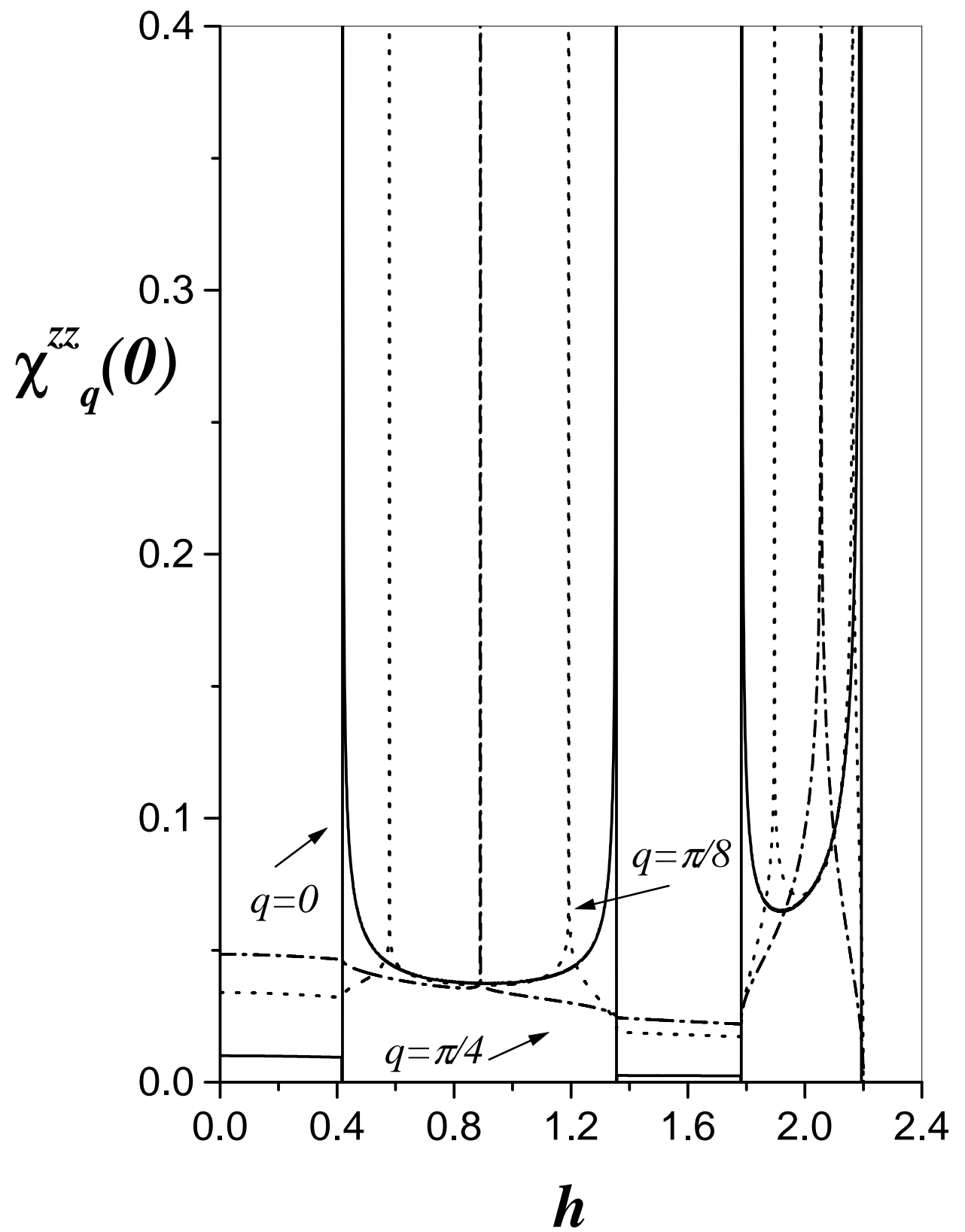

Fig. 8. Static susceptibility in the field direction, $\chi_{q}^{z z}(0)$, at $T=0$, as a function of the field for $n=4, J_{1}=1, J_{2}=2, J_{3}=1.5, J_{4}=5 / 3, \mu_{1}=1, \mu_{2}=0.5, \mu_{3}=1$, $\mu_{4}=0.5$ and different values of $q$. 

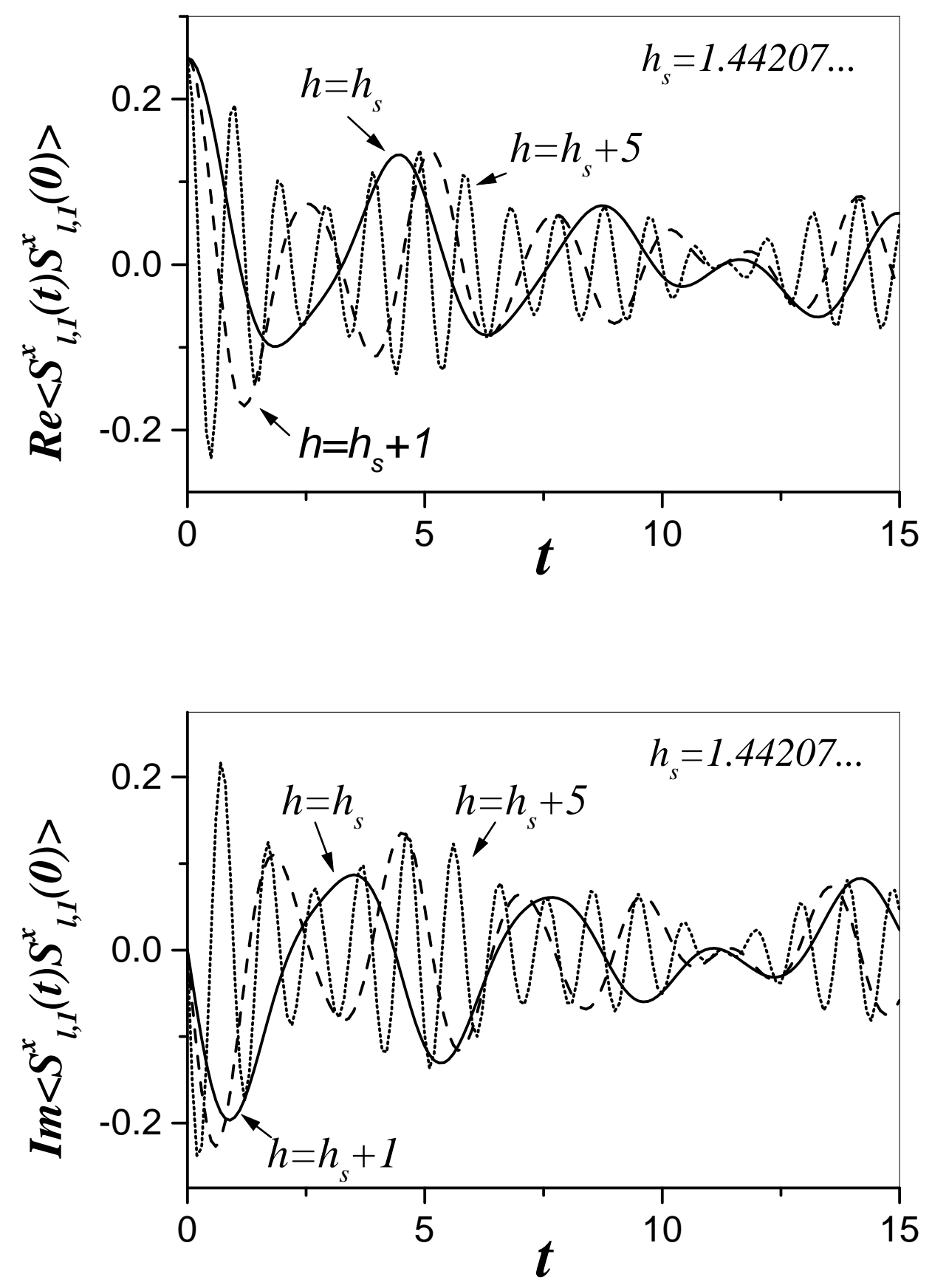

Fig. 9. The real and imaginary parts of the correlation function $\left\langle S_{l, 1}^{x}(t) S_{l, 1}^{x}(0)\right\rangle$, as functions of time, at $T=0$, for $n=8, J_{1}=1, J_{2}=2, J_{3}=1.5, J_{4}=5 / 3, J_{5}=2 / 3$, $J_{6}=3 / 5, J_{7}=2, J_{8}=1, \mu_{1}=\ldots=\mu_{8}=1$, for values of the field at and at above the saturation field $h_{s}$. 

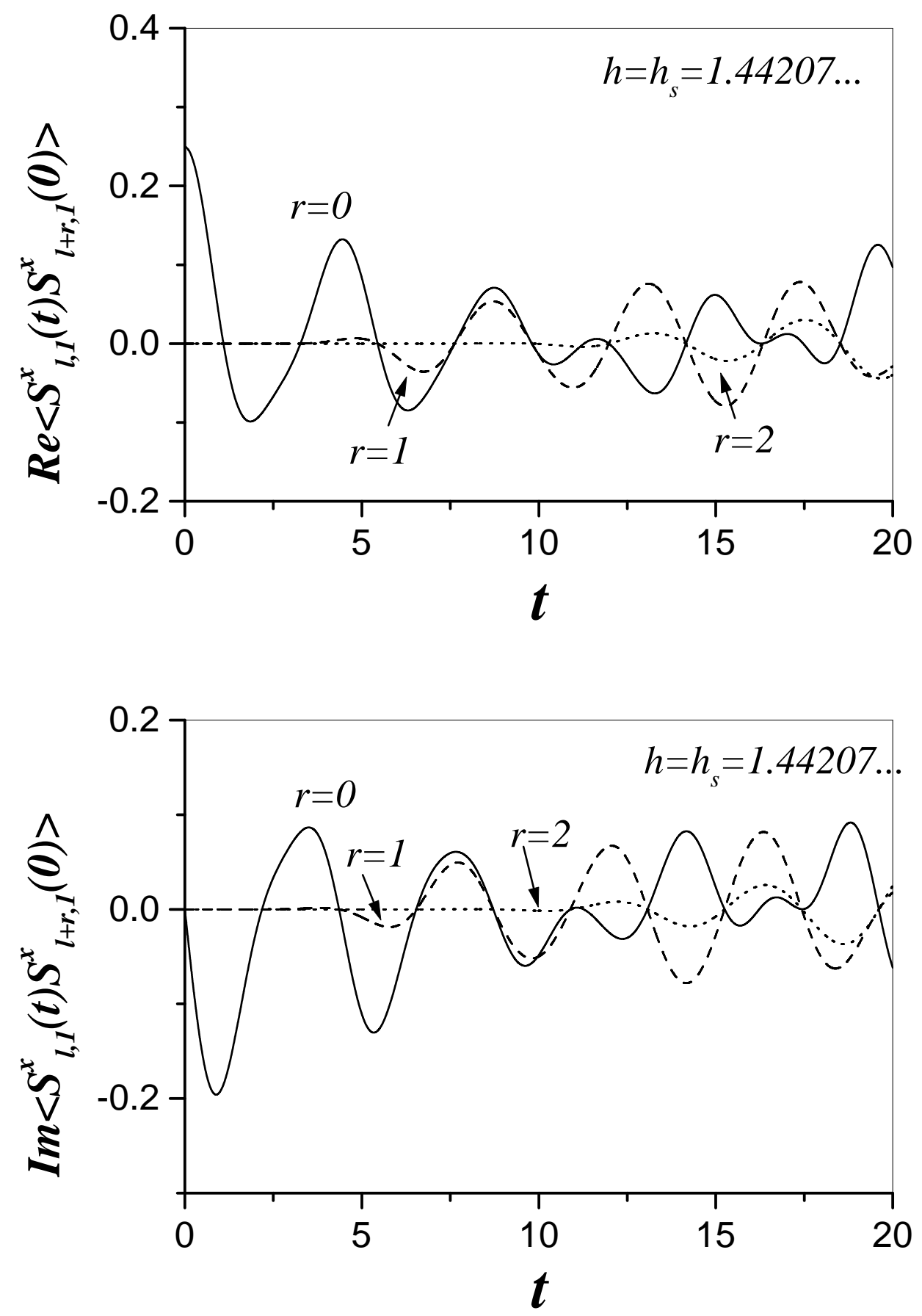

Fig. 10. The real and imaginary parts of the correlation function $\left\langle S_{l, 1}^{x}(t) S_{l+r, 1}^{x}(0)\right\rangle$, as functions of time, at $T=0$, for $n=8, J_{1}=1, J_{2}=2, J_{3}=1.5, J_{4}=5 / 3$, $J_{5}=2 / 3, J_{6}=3 / 5, J_{7}=2, J_{8}=1, \mu_{1}=\ldots=\mu_{8}=1$, at the saturation field $h_{s}$. 
(a)

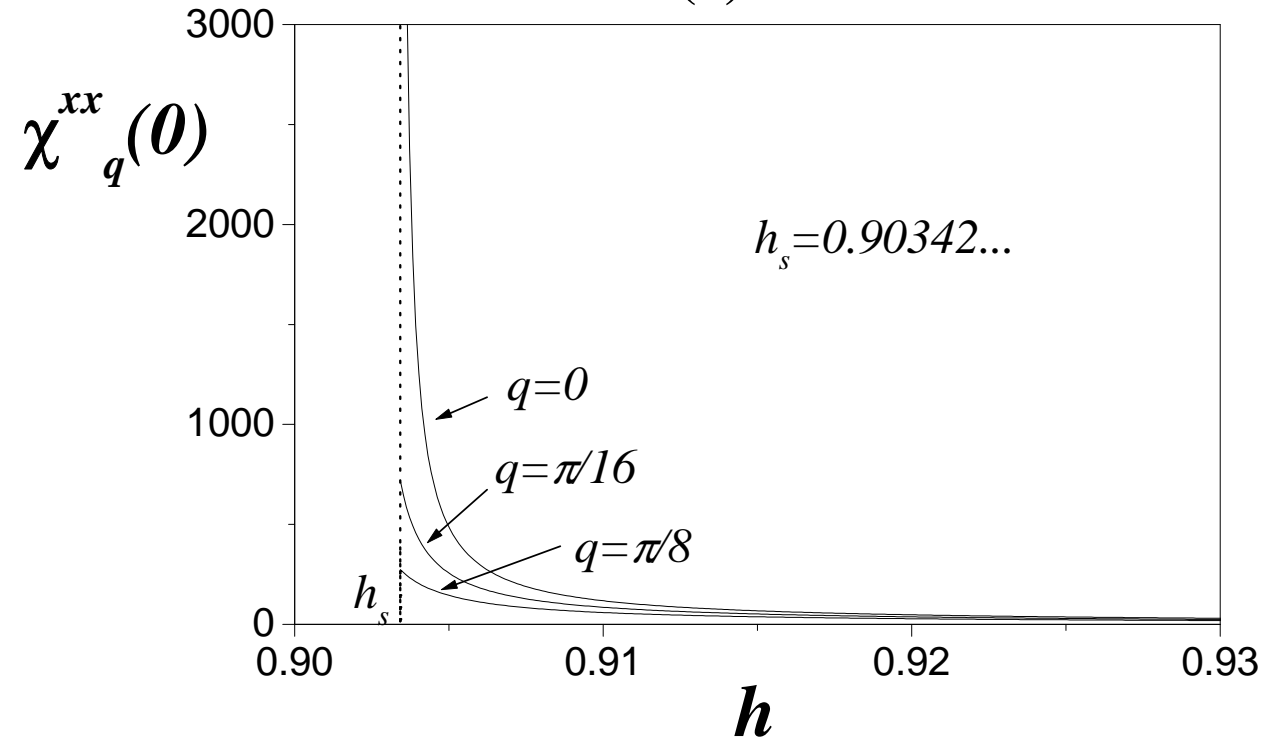

(b)

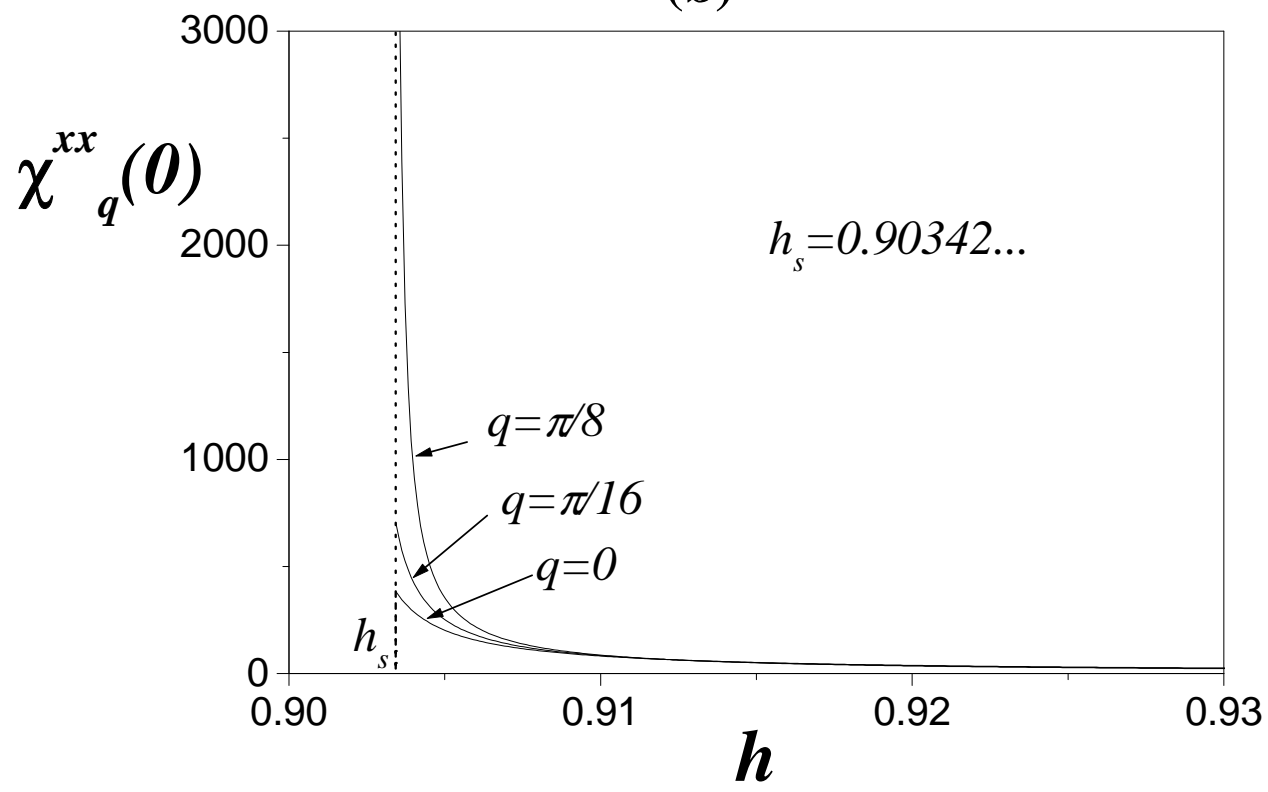

Fig. 11. Static susceptibility $\chi_{q}^{x x}(0)$ as a function of the field, at $T=0$, for $n=8$, $\mu_{1}=3, \mu_{2}=4, \mu_{3}=\mu_{5}=\mu_{7}=1.5, \mu_{4}=2.5, \mu_{6}=\mu_{8}=1$, and different values of $q$. (a) Corresponds to $J_{1}=1, J_{2}=2, J_{3}=1.5, J_{4}=5 / 3, J_{5}=2 / 3, J_{6}=3 / 5$, $J_{7}=2, J_{8}=1$ and (b) $J_{1}=-1, J_{2}=2, J_{3}=1.5, J_{4}=5 / 3, J_{5}=2 / 3, J_{6}=3 / 5$, $J_{7}=2, J_{8}=1$. 
which is equivalent to the eigenvalue equation shown in eq.(14). From this set of equations we can write the matrix equation

$$
\left(\begin{array}{c}
A_{q, m+1} \\
A_{q, m}
\end{array}\right)=\mathbb{T}_{m}(\omega, h)\left(\begin{array}{c}
A_{q, m} \\
A_{q, m-1}
\end{array}\right), \text { for } m=2,3, \ldots, n-1,
$$

where

$$
\mathbb{T}_{m}(\omega, h) \equiv\left(\begin{array}{cc}
-2\left(\frac{\omega+\mu_{m} h}{J_{m}}\right) & -\frac{J_{m-1}}{J_{m}} \\
1 & 0
\end{array}\right)
$$

and

$$
\begin{aligned}
\left(\begin{array}{l}
A_{q, 2} \\
A_{q, 1}
\end{array}\right) & =\widetilde{\mathbb{T}}_{1}(\omega, h)\left(\begin{array}{c}
A_{q, 1} \\
A_{q, n}
\end{array}\right) \text { for } m=1, \\
\left(\begin{array}{c}
A_{q, 1} \\
A_{q, n}
\end{array}\right) & =\widetilde{\mathbb{T}}_{n}(\omega, h)\left(\begin{array}{c}
A_{q, n} \\
A_{q, n-1}
\end{array}\right), \text { for } m=n
\end{aligned}
$$

with $\widetilde{\mathbb{T}}_{1}$ and $\widetilde{\mathbb{T}}_{n}$ given by

$$
\begin{aligned}
& \widetilde{\mathbb{T}}_{1}(\omega, h) \equiv\left(\begin{array}{cc}
-2\left(\frac{\omega+\mu_{1} h}{J_{1}}\right) & -\frac{J_{n}}{J_{1}} \exp (i d q) \\
1 & 0
\end{array}\right) \\
& \widetilde{\mathbb{T}}_{n}(\omega, h) \equiv \exp (i d q)\left(\begin{array}{cc}
-2\left(\frac{\omega+\mu_{n} h}{J_{n}}\right) & -\frac{J_{n-1}}{J_{n}} \\
\exp (-i d q) & 0
\end{array}\right)
\end{aligned}
$$

which satisfy the result,

$$
\widetilde{\mathbb{T}}_{1}(\omega, h) \widetilde{\mathbb{T}}_{n}(\omega, h)=\exp (i n q) \mathbb{T}_{1}(\omega, h) \mathbb{T}_{n}(\omega, h)
$$

with $\mathbb{T}_{1}$ and $\mathbb{T}_{n}$ given by

$$
\begin{aligned}
& \mathbb{T}_{1}(\omega, h) \equiv\left(\begin{array}{cc}
-2\left(\frac{\omega+\mu_{1} h}{J_{1}}\right) & -\frac{J_{n}}{J_{1}} \\
1 & 0
\end{array}\right), \\
& \mathbb{T}_{n}(\omega, h) \equiv\left(\begin{array}{cc}
-2\left(\frac{\omega+\mu_{n} h}{J_{n}}\right) & -\frac{J_{n-1}}{J_{n}} \\
1 & 0
\end{array}\right),
\end{aligned}
$$


From eqs.(A.5-A.13) we can write

$$
\mathbb{T}_{\text {cell }}(\omega, h)\left(\begin{array}{c}
A_{q, 2} \\
A_{q, 1}
\end{array}\right)=\exp (-i q d)\left(\begin{array}{c}
A_{q, 2} \\
A_{q, 1}
\end{array}\right) \equiv \exp (-i q d) \Psi_{q, 1,2}
$$

where

$$
\mathbb{T}_{\text {cell }}(\omega, h) \equiv \mathbb{T}_{1}(\omega, h) \mathbb{T}_{n}(\omega, h) \mathbb{T}_{n-1}(\omega, h) \mathbb{T}_{n-2}(\omega, h) \ldots \mathbb{T}_{3}(\omega, h) \mathbb{T}_{2}(\omega, h)
$$

Eq.(A.14) shows explicitly that $\exp (-i q n)$ is an eigenvalue of $\mathbb{T}_{\text {cell }}(\omega, h)$ corresponding to the eigenvector $\Psi_{q, 1,2}$. Since $\mathbb{T}_{\text {cell }}(\omega, h)$ does not depend on $q$, we can obtain immediately the second eigenvector of $\mathbb{T}_{\text {cell }}(\omega, h)$, and the respective eigenvalue, from this equation by introducing the transformation $q \rightarrow-q$, which gives $\Psi_{-q, 1,2}$ and $\exp (-i q d)$ respectively. These results are consistent with the fact that $\operatorname{det}\left[\mathbb{T}_{\text {cell }}(\omega, h)\right]=1$, and we can write finally the equation

$$
\operatorname{trace}\left[\mathbb{T}_{\text {cell }}(\omega, h)\right]=2 \cos (d q),
$$

whose solution will give the excitation spectrum.

In the absence of the external field, for $n$ odd, we can show that the previous equation can be written in the form

$$
f(\omega) \times \omega=2 \cos (d q),
$$

where $f(\omega)$ is a polynomial function of degree $n-1$. This means that $q=\pi / 2 d$ is a zero-energy mode irrespective of the values of $J^{\prime} s$.

On the other hand, for $n$ even and zero external field, eq.(A.16) can be written as

$$
g(\omega) \times \omega^{2}+(-1)^{n / 2}\left[\frac{J_{2} J_{4} \ldots J_{n}}{J_{1} J_{3} \ldots J_{n-1}}+\frac{J_{1} J_{3} \ldots J_{n-1}}{J_{2} J_{4} \ldots J_{n}}\right]=2 \cos (d q),
$$

where $g(\omega)$ is a polynomial of degree $n-2$. From this result we can conclude that $q=0$ is a zero-energy mode provided the condition $J_{1} J_{3} \ldots J_{n-1}=$ $J_{2} J_{4} \ldots J_{n}$ is satisfied. Therefore, under this condition, there is no energy gap between the ground state and the first excited state in the absence of external field. 


\section{B Dynamic correlation $\left\langle S_{l, m}^{x}(t) S_{l+r, m^{\prime}}^{x}(0)\right\rangle$}

The dynamic correlation function $\left\langle S_{l, m}^{x}(t) S_{l+r, m^{\prime}}^{x}(0)\right\rangle$, in terms of the hamiltonians $H^{ \pm}$, in the thermodynamic limit, can be written in the form $[20,21,22]$

$$
\left\langle S_{l, m}^{x}(t) S_{l+r, m^{\prime}}^{x}(0)\right\rangle=\frac{\operatorname{Tr}\left[\exp \left(-\beta H^{-}\right) \exp \left(i H^{-} t\right) S_{l, m}^{x} \exp \left(-i H^{+} t\right) S_{l+r, m^{\prime}}^{x}\right]}{\operatorname{Tr}\left[\exp \left(-\beta H^{-}\right)\right]},
$$

which is very difficult to calculate, since $H^{-}$and $H^{+}$do not commute. However, at $T=0$, for external field greater than the saturation field $\left(h \geqslant h_{s}\right)$, $\mathrm{H}^{+}$and $\mathrm{H}^{-}$have identical ground state, namely,

$$
\left|\Phi_{0}\right\rangle=\prod_{l, m} \otimes\left|n_{l m}\right\rangle
$$

where $n_{l m}=1, \forall l, m$, and

$$
H^{-}\left|\Phi_{0}\right\rangle=H^{+}\left|\Phi_{0}\right\rangle=E_{0}\left|\Phi_{0}\right\rangle .
$$

Then, in this limit, the dynamic correlation $\left\langle S_{1, m}^{x}(t) S_{1+r, m^{\prime}}^{x}(0)\right\rangle$ is given by

$$
\left\langle S_{1, m}^{x}(t) S_{1+r, m^{\prime}}^{x}(0)\right\rangle=\left\langle\Phi_{0}\left|\exp \left(i H^{+} t\right) S_{1, m}^{x} \exp \left(-i H^{-} t\right) S_{1+r, m^{\prime}}^{x}\right| \Phi_{0}\right\rangle .
$$

From the eqs.(2) and (3) we can express $S_{l, m}^{x}$ in terms of fermion operators in the form

$$
S_{l, m}^{x}=\frac{1}{2} \exp \left\{i \pi\left[\sum_{j=1}^{r} \sum_{k=1}^{n} c_{j, k}^{\dagger} c_{j, k}+\sum_{k^{\prime}=1}^{m^{\prime}-1} c_{1+r, k^{\prime}}^{\dagger} c_{1+r, k^{\prime}}\right]\right\}\left(c_{1+r, m^{\prime}}^{\dagger}+c_{1+r, m^{\prime}}\right),
$$

and substituting this result in eq.(B.4) we obtain

$$
\begin{aligned}
\left\langle S_{1, m}^{x}(t) S_{1+r, m^{\prime}}^{x}(0)\right\rangle= & \frac{(-1)^{n r+m^{\prime}-1}}{4} \exp \left(i E_{0} t\right)(-1)^{m-1}\left\langle\Phi_{0}\right|\left(c_{1, m}^{\dagger}+c_{1, m}\right) \times \\
& \times \exp \left(-i H^{-} t\right)\left(c_{1+r, m^{\prime}}^{\dagger}+c_{1+r, m^{\prime}}\right)\left|\Phi_{0}\right\rangle,
\end{aligned}
$$

which can be written as

$$
\begin{aligned}
\left\langle S_{1, m}^{x}(t) S_{1+r, m^{\prime}}^{x}(0)\right\rangle= & \frac{(-1)^{n r+m^{\prime}-m}}{4}\left\langle\Phi_{0}\right| \exp \left(i H^{-} t\right)\left[c_{1, m}^{\dagger}+c_{1, m}\right] \exp \left(-i H^{-} t\right) \times \\
& \times\left[c_{1+r, m^{\prime}}^{\dagger}+c_{1+r, m^{\prime}}\right]\left|\Phi_{0}\right\rangle .
\end{aligned}
$$


Finally, by using eq.(63), we obtain

$$
\left\langle S_{1, m}^{x}(t) S_{1+r, m^{\prime}}^{x}(0)\right\rangle=\frac{(-1)^{n r+m^{\prime}-m}}{4 N} \sum_{q, k} \exp (-i q d r) u_{q, k m}^{*} u_{q, k m^{\prime}} \exp \left(i \varepsilon_{q, k} t\right),
$$

which reduces to the known result for the homogeneous chain [38].

From the previous equation we can conclude immediately that the static correlation $\left\langle S_{1, m}^{x} S_{1+r, m^{\prime}}^{x}\right\rangle$ is equal to $\delta_{q, 0} / 4$

\section{References}

[1] E. Dagotto and T. M. Rice, Surprises on the way from one- to two-dimensional quantum magnets: the ladder materials, Science 271 (1996) 618-623.

[2] T. N. Nguyen, P. A. Lee and H. C. Loye, Design of a random quantum spin chain paramagnet: $\mathrm{Sr}_{3} \mathrm{CuPt} \mathrm{t}_{0.5} \mathrm{Ir}_{0.5} \mathrm{O}_{6}$, Science 271 (1996) 489-491.

[3] P. Gambardella, A. Dallmeyer, K. Maiti, M. C. Malagoli,W. Eberhardt, K. Kern and C. Carbone, Ferromagnetism in one-dimensional monoatomic metal chains, Nature 416 (2002) 301-303.

[4] C.J. Mukherjeea, R. Coldeaa, D.A. Tennanta, M. Kozac, M. Enderlec, K. Habichtd, P. Smeibidld, and Z. Tylczynskie, Field-induced quantum phase transition in the quasi $1 \mathrm{D}$ XY-like antiferromagnet $C s_{2} C o C l_{4}, J$. Magn. Magn. Matt, 272-276 (2004) 920-921.

[5] S. Sachdev, Quantum phase transitions (Cambridge Univ. Press, 2000).

[6] K. Okamoto, Level spectroscopy: physical meaning and application to the magnetization plateau problems, Prog. Theoret. Phys. Supp. 145 (2002) 113118.

[7] I. G. Bostrem, A. S. Boyarchenkov, A. A. Konovalov, A. S. Ovchinnikov and V. E. Sinitsyn, J. Exp. and Theoret. Phys. 97 (2003) 615-623.

[8] S. Kramp, M. Loewenhaupt and M. Rotter, The spin wave dispersion of $N d C u 2$ in strong magnetic fields, Physica B, 276-278 (2000) 628-629.

[9] P. Coleman, Magnetic spins that last for ever, Nature 413 (2001) 788-789.

[10] M. Matsumoto, B. Normand, T. M. Rice and M. Sigrist, Field- and pressureinduced magnetic quantum phase transitions in $T l C u C l_{3}$, Phys. Rev. B 69 (2004) 054423.

[11] H. E. Lieb, T. Schultz and D. C. Mattis, Two soluble models of antiferromagnetic chain, Ann. Phys. 16 (1961) 407-466. 
[12] O. Derzhko, J. Richter, T. Krokhmalskii and O. Zaburannyi, Regularly alternating spin-1/2 anisotropic XY chains: The ground-state and thermodynamic properties, Phys.Rev.E 69 (2004) 066112.

[13] O. Derzhko, J. Richter and O. Zaburannyi, Thermodynamic properties of the periodic nonuniform spin-1/2 isotropic XY chains in a transverse field, Physica A 282 (2000) 495-524.

[14] V. M. Kontorovich and V. M. Tsukernik, Magnetic properties of a spin array with two sublattices, Sov. Phys. JETP 26 (1968) 687-691.

[15] J. H. H. Perk and H. W. Capel, Time- and frequency-dependent correlation functions for the homogeneous and alternating isotropic XY-models, Physica 100A (1980) 1-23.

[16] O. Derzhko, T. Krokhmalskii and J. Stolze, Dynamics of the spin-1/2 isotropic XY chain in a transverse field, J. Phys. A: Math. Gen. 33 (2000) 3063-3080.

[17] J. P. de Lima and L. L. Gonçalves, The longitudinal dynamic correlation and dynamic susceptibility of the isotropic XY-model on the 1D alternating superlattice, Physica A 311 (2002) 458-474.

[18] J. P. de Lima and L. L. Gonçalves, The XY model on the one-dimensional superlattice: static properties, J. Magn. Magn. Mater. 206 (1999) 135-148.

[19] L. L. Gonçalves and J. P. de Lima, The XY-model on the one-dimensional superlattice, J. Magn. Magn. Mater. 140-144 (1995) 1606-1608.

[20] Th. J. Siskens and P. Mazur, Time-correlation functions in the a-ciclic XY model, Physica 71 (1974) 560-578.

[21] H. W. Capel and J. H. H. Perk, Autocorrelation function of x-component of the magnetization in the one-dimensional XY-model, Physica A 87 (1977) 211-242.

[22] L. L. Goncalves, Theory of properties of some one-dimensional systems (D. Phil. Thesis, University of Oxford, 1977).

[23] F. F. Barbosa Filho, J. P. de Lima and L. L. Gonçalves, The anisotropic XY model on the 1D alternating superlattice, J. Magn. Magn. Mater. 226-230 (2001) 638-640.

[24] G. A. Korn and T. M. Korn, Mathematical handbook for scientists and engineers (Mc Graw-Hill, New York, 1961).

[25] P. Pfeuty, An exact result for the 1D random Ising model in a transverse field, Phys. Lett. A 72 (1979) 245-246.

[26] R. Jullien and J. N. Fields, Equivalence between a spin 1/2 dimerized XY chain and two independent Ising chains in transverse fields, Phys. Lett. A 69 (1978) 214-216.

[27] D. S. Fisher, Random antiferromagnetics quantum spins chains, Phys. Rev. B 50 (1994) 3799-3821. 
[28] M. Oshikawa, M. Yamanaka and I. Affleck, Magnetization plateaus in spin chains: "Haldane gap" for half-integer spins, Phys. Rev. Lett. 78 (1997) 19841987.

[29] Th. Niemeijer, Some exact calculations on a chain of spin 1/2, Physica 37 (1967) 377-419.

[30] W. E. Parry, The many-body problem (Oxford University Press, Oxford, 1973).

[31] D. N. Zubarev, Double-time Green functions in statistical physics, Sov. Phys. Usp. 3 (1960) 320-345.

[32] S. Katsura and T. Horiguchi, Dynamic properties of the isotropic XY model, Physica 46 (1970) 67-86.

[33] R. Jullien and P. Pfeuty, Zero-temperature renormalization-group method for quantum systems. II. Isotropic $\mathrm{X}-\mathrm{Y}$ model in a transverse field in one dimension, Phys. Rev B 19 (1979) 4646-4652.

[34] L. L. Gonçalves, Transverse susceptibility of 1D isotropic XY-model at zero temperature, Rev. Bras. Fis. 16 (1986) 491-494.

[35] T. Horiguchi and T. Morita, Isothermal and frequency dependent perpendicular susceptibilities of the Ising model on the Cayley tree, Can. J. Phys. 53 (1975) 2375-2386.

[36] H. E. Stanley, Phase transitions and critical phenomena (Oxford University Press, Oxford, 1971).

[37] J. P. de Lima and L. L. Gonçalves, Surface tension of the induced magnetization in the 1D isotropic XY-model, Mod. Phys. Lett. B 11 (1997) 9-15.

[38] H. B. Cruz and L. L. Gonçalves, Time-dependent correlation of the onedimensional isotropic XY model, J. Phys. C 14 (1981) 2785-2791 